\title{
The Perron-Frobenius operator in spaces of smooth functions on an interval
}

\author{
B. SZEWC \\ SGGW-Warsaw Agricultural University, Institute of Applied Mathematics \\ and Statistics, Warsaw, Poland
}

(Received 14 March 1983 and revised 12 January 1984)

\begin{abstract}
The densities of invariant measures for Misiurewicz maps and LasotaYorke maps of class $C^{r}$ are of class $C^{r-1}$ on certain intervals (forming the partition of an interval in case of Misiurewicz maps). For these maps the Perron-Frobenius operator has an unambiguous decomposition into the sum of projections onto eigenspaces (multiplied by the eigenvalues) and a remainder operator. The remainder operator has spectral radius less than one in certain spaces of smooth functions.
\end{abstract}

\section{Introduction}

A. Lasota and J. Yorke [5] started the long series of studies of ergodic properties of piecewise monotonic transformations of an interval with derivative greater than one. Advanced research in this domain is found in [1] which is written in terms of functional analysis. M. Rychlik [8] has obtained similar results for maps with a countable number of pieces of monotonicity and without the application of the strong Ionescu-Tulcea, Marinescu theorem.

Other useful references have maps with singularities where the derivative is equal to zero (for example the famous family of quadratic maps $\{\alpha x(1-x)\}$ ). I recall only the papers closely related to the present research. M. Misiurewicz [6] has proved the existence and studied properties of absolutely continuous invariant measures for negative Schwarzian maps without sinks and such that the set of critical points is separated from the trajectory. W. Szlenk [10] has proved the existence of absolutely continuous measures for similar maps which are not necessarily negative Schwarzian.

The third subject connected with this research is the question of smoothness of densities of invariant measures. This question for expanding maps was answered by $R$. Sacksteder [9] (unfortunately the paper contains an important mistake) and K. Krzyżewski [4] who proved that if an expanding map is of class $C^{r}$ then the density is of class $C^{r-1}$.

In this paper we construct the spaces $C_{f, \varepsilon}^{r-1}$ of functions of class $C^{r-1}$ on intervals forming a partition with a weighted sup-norm.

The Perron-Frobenius operator for Lasota-Yorke maps or Misiurewicz maps has an unambiguous decomposition into the sum of projections onto the eigenspaces and the remainder operator. The spectral radius of the remainder operator in the 
space $C_{f, \varepsilon}^{r-1}$ is less than one. Proving this we also show that the densities of invariant measures belong to $C_{f, \varepsilon}^{r-1}$.

The question of convergence of mixing coefficients also appears to be connected with the work in this paper. We recall that if they converge to zero exponentially, then the map satisfies the Central Limit Theorem. K. Ziemian [11] applied theorem 6.3(d) in the proof that for Misiurewicz maps mixing coefficients tend to zero exponentially. F. Hofbauer and G. Keller [2, paragraph 4] have proved this for the maps considered in [10] when critical points fall into the repelling periodic orbits.

$\$ 1$ contains the lemmas which allow us to estimate the derivatives of images of functions under the Perron-Frobenius operator.

$\S \S 2-5$ contain definitions, lemmas and propositions about Misiurewicz maps which are necessary to prove the main result theorem 6.3 . Thus in $\S 2$ we define the class $\mathcal{M}$ of mappings and give estimates of distortion and of derivatives of iterates of a map. $\S 3$ gives the estimates of images of functions under the Perron-Frobenius operator and auxiliary operators $f_{(i)}$ far from the trajectories of the critical points. $\$ 4$ defines the class $\mathcal{M}^{r}$ of maps (of class $C^{r}$ ) and Banach spaces $C_{f, \varepsilon}^{s}$ and extends results of $\S 3$. In $\S 5$ we prove the fundamental inequalities (proposition $5.4(\mathrm{~b})$ ), which suggest a possibility of an application of Ionescu-Tulcea, Marinescu theorem. Unfortunately we don't know how to secure a compactness property and therefore we prove theorem 6.3 in a way similar to that of K. Krzyżewski.

In $\S 6$ we reformulate certain results of $M$. Misiurewicz [6] and prove the main theorem about maps in the class $\mathscr{M}^{r}$. At the end we prove the spectral decomposition of the Perron-Frobenius operator for maps in the class $\mathcal{M}$.

$\S 7$ is devoted to Lasota-Yorke maps. It contains two different definitions of Banach spaces of differentiable functions (the second one does not depend on the map). We state there that the densities of invariant measures belong to this space and the spectral radius of the remainder operator is less than one.

In what follows we assume that the reader is familiar with the paper of $M$. Misiurewicz [6].

Our results here answer the question about a class of smoothness of invariant measures posed to me by W. Szlenk. The general question about analyticity of invariant measures for analytic maps remains open. For Misiurewicz or Szlenk maps given by a real polynomial the answer is positive. It is proved by the reasoning contained at the beginning of [7] applied to these maps.

After I had written this paper, I found that the condition (iii) (see $\S 2$ ) on the Schwarzian derivative can be replaced by a weaker one as follows:

(iii') There exists $\delta$ such that $W_{\delta}$ satisfies the conclusion of theorem $2.1, W_{\delta} \subset \mathcal{U}$ (see (v) and (2.0) of $\S 2$ ) and if $x \in W_{\delta}$ and $f^{k}(x) \in W_{\delta}$ then $\left|\left(f^{k}\right)^{\prime}(x)\right|>1$.

(A similar condition was suggested by W. Szlenk.)

I conjecture that this condition also allows us to prove all the main results of $M$. Misiurewicz [6] and all the results of the present paper. Moreover in this case the map could be of class $C^{2}$ instead of $C^{3}$ and it would be possible to treat jointly Misiurewicz maps and Szlenk maps.

When I was finishing this paper I learned that K. Bugiel at Jagiellonian University had obtained results similar to those in $\$ 7$. 
I would like to thank W. Szlenk for discussions which allowed me to avoid several mistakes and M. Rychlik for talks about Lasota-Yorke type maps. I am also grateful to $\mathrm{K}$. Ziemian for help in preparing the present version of the paper.

Now we introduce the notation.

We shall consider in $\S \S 2-7$ maps $f: I \backslash A \rightarrow I$, where $I$ is an interval, $A \subset I$ a finite set containing the endpoints of $I$. We will assume that $f$ is continuous and monotonic on components of $I \backslash A$. Write

$$
\hat{I}=I \times\{+,-\} \backslash\{(\text { left endpoint of } I,-) \text {, (right endpoint of } I,+)\} \text {. }
$$

We shall pretend that $f$ acts on $\hat{I}$ (i.e. $f: \hat{I} \rightarrow \hat{I}$ ) as follows: $f(x, \varepsilon)=\left(x^{\prime}, \varepsilon^{\prime}\right)$, where $x^{\prime}$ is the limit of $f(y)$ as $y$ tends to $x$ from the right if $\varepsilon=+$, (left if $\varepsilon=-$ ) and $\varepsilon^{\prime}=\varepsilon$ if and only if $f$ preserves the orientation in a right-neighbourhood of $x$ if $\varepsilon=+$, (left if $\varepsilon=-$ ). If $x \in \hat{I}$ then $\check{x}$ is its first coordinate and $\underline{x}$ the second one. We suggest the reader thinks of $(x,+)$ as $x$ with its right neighbourhood and $(x,-)$ as $x$ with its left neighbourhood. Then $f((x,+))$ indicates whether the right neighbourhood of $x$ is mapped to the right or to the left of $f((x,+))^{\vee}$. This notation gives an advantage in (3.10), where we define the weight functions $\varphi_{i}$. It also enables us to treat jointly the cases of two- and one-sided derivatives in $\S \S 3-5$. We set

$$
\begin{gathered}
\hat{A}=\hat{I} \cap(A \times\{+,-\}), \quad \hat{B}_{n}=\bigcup_{k=1}^{n} f^{k}(\hat{A}), \\
\hat{B}=\hat{B}_{+\infty}, \quad B_{n}=\left(\hat{B}_{n}\right)^{\vee}, \quad B=(\hat{B})^{\vee}, \\
\hat{A}_{1}=\hat{A} \cap \hat{B}, \quad \hat{A}_{2}=\hat{A} \backslash \hat{A}_{1} .
\end{gathered}
$$

For $x \in I$ and $y \in \hat{I}$ define the distance

$$
\operatorname{dist}(x, y)= \begin{cases}|x-\check{y}| & \text { iff either } \underline{y}=+ \text { and } \check{y}<x \text { or } \underline{y}=- \text { and } \check{y}>x, \\ +\infty & \text { otherwise. }\end{cases}
$$

Then for $x \in I$ and $D \subset \hat{I}$, dist $(x, D)=\inf _{y \in D} \operatorname{dist}(x, y)$. We also define a one-sided $\varepsilon$-neighbourhood. For $x \in \hat{I}$ and $\varepsilon>0$,

$$
F(x, \varepsilon)=\{y \in I: \operatorname{dist}(y, x)<\varepsilon\}= \begin{cases}] \check{x}, \check{x}+\varepsilon[ & \text { if } \underline{x}=+ \\ ] \check{x}-\varepsilon, \check{x}[ & \text { if } \underline{x}=-.\end{cases}
$$

Let $g: V \rightarrow \mathbb{R}$ be a map $(V \subset I)$, then for $x \in \hat{I}, g^{(s)}(x)$ will denote the (left-sided if $\underline{x}=-$, right-sided if $\underline{x}=+$ ) sth derivative at $x, s \in \mathbb{N}$. $\lambda$ will be Lebesgue measure on $I$.

The main results of this paper are proved in $\S 6$. For their proofs, the results of [6] and those from $\S \S 1-5$ of this paper, only proposition 5.4 (together with remark 5.5) and remark 4.3 are necessary. The proof of remark 4.3 is independent of the other results of this paper. In order to help the reader understand the course of the proof of proposition 5.4 we give the following short description of it:

Fix some $n$ and a $\delta$ which is small enough. We write $W$ for $W_{\delta}$, where $W_{\delta}$ is some neighbourhood of critical points (see (2.0)). Set

$$
\begin{aligned}
& U_{i}=\left\{x: f^{i-1}(x) \in W, f^{j}(x) \notin W \quad \text { for } i \leq j \leq n-1\right\} \quad 1 \leq i \leq n ; \\
& E_{k}=\left\{x: f^{j}(x) \notin W \quad \text { for } 0 \leq j \leq k-1\right\} \quad 0 \leq k \leq n ; \\
& U_{0}=E_{n} .
\end{aligned}
$$


We have $f^{i}\left(U_{i}\right) \subset E_{n-i}$. Hence, since $(f+g)_{*}=f_{*}+g_{*}$ and $(f \circ g)_{*}=f_{*} \circ g_{*}$, where $f_{*}$ is defined in (1.6), we have

$$
f_{*}^{n}(\rho)=\sum_{j=0}^{n}\left(\left.f^{n-j}\right|_{E_{n-j}}\right)_{*} \circ\left(\left.f^{j}\right|_{U_{j}}\right)_{*}(\rho)
$$

Define $\|\rho\|=\sup _{x \in I}|\rho(x)| / \varphi(x)$ for some weight function $\varphi$. We can then show for $\xi(a)=u(a) /(1+u(a))$ that there is an $H>0$ with (lemma 5.3):

$$
\left|\left(f^{j} / U_{j}\right)_{*}(\rho)\right| \leq H\|\rho\| \sum_{a \in \hat{A}_{2}} \psi_{f(a)}^{\xi(a)}
$$

where $\psi_{b}$ is defined in (3.1), and that for $\eta \in(0,1)$ there are $G>0$ and $h \in(0,1)$ with

$$
\left(\left.f^{k}\right|_{E_{k}}\right)_{*}\left(\psi_{b}^{\eta}\right) \leq G\left(\left|\left(f^{k}\right)^{\prime}(b)\right|^{\eta-1} \psi_{f^{k}(b)}^{\eta}+h^{k}\right)
$$

(lemma 5.1 for $i=0$ ) where $b \in \hat{B}$ and $k \in \mathbb{N}$. Putting (2) and (3) into (1) we get

$$
\begin{aligned}
\left|f_{*}^{n}(\rho)\right| & \leq H\|\rho\| \sum_{j=0}^{n} \sum_{a \in \hat{A}_{2}}\left(G\left|\left(f^{n-j}\right)^{\prime}(f(a))\right|^{\xi(a)-1} \psi_{f^{n-j+1}(a)}^{\xi(a)}+h^{n-j}\right) \\
& \leq C\|\rho\|\left(\sum_{m=0}^{+\infty} \sum_{a \in \hat{A}_{2}}\left|\left(f^{m}\right)^{\prime}(f(a))\right|^{\xi(a)-1}\left(\psi_{f^{m+1}(a)}^{\xi(a)}\right)+1\right)=C\|\rho\| \varphi
\end{aligned}
$$

and we have an estimate of $\left\|f_{*}^{n}(\rho)\right\|$. This explains the choice of the weight function $\varphi$.

In order to establish (2), we need the existence of $G$ with

$$
\left|f_{*}^{n}(\rho)\right| \leq G\|\rho\| \quad \text { on } W .
$$

Because $|\rho| \leq \varphi\|\rho\|$ (cf. definition of $\|\cdot\|$ ) we have (this shows the advantage of defining $\|\cdot\|$ as a weighted sup-norm):

$$
\left|f_{*}^{n}(\rho)\right| \leq f_{*}^{n}(|\rho|) \leq f_{*}^{n}(\varphi\|\rho\|)=\|\rho\| f_{*}^{n}(\varphi),
$$

so that it suffices to show

$$
f_{*}^{n}(\varphi) \leq G \varphi \quad \text { on } W \text {. }
$$

As $\varphi$ is a linear combination of $\psi_{b}^{\eta}$ and 1, it suffices to prove (5) for these functions instead of $\varphi$. This is done in lemma 3.1.

But we also want to estimate derivatives of $f_{*}^{n}(\rho)$, so we start in $\S 1$ with the proof of

$$
\left|f_{*}^{n}(\rho)^{(s)}(x)\right| \leq \sum_{i=0}^{s} f_{(i)}^{n}\left(\left|\rho^{(i)}\right|\right)(x) \cdot T(x)
$$

where

$$
f_{(i)}(\rho)(x)=\sum_{y \in f^{-1}(x)} \rho(y)\left|f^{\prime}(y)\right|^{-i-1},
$$

and the function $T$ is bounded on $W$ which is proved in $\S 4$. As above we define semi-norms for the derivatives

$$
|\rho|_{i}=\sup _{I} \frac{\left|\rho^{(i)}\right|}{\varphi_{i}} \text { and }|\rho|_{1, s}=\max \left\{|\rho|_{1}, \ldots,|\rho|_{s}\right\}
$$

where $\varphi_{i}$ is again a suitable weight function. In order to estimate $\left|f_{*}^{n}(\rho)\right|_{1, s}$ we use the derivatives of (1). We have to apply (6) for $\left(\left(\left.f^{n-j}\right|_{E_{n-i}}\right)_{*}\left(\tilde{\rho}_{j}\right)\right)^{(s)}$ with $\tilde{\rho}_{j}=$ 
$\left(f^{j} / U_{j}\right)_{*}(\rho)$, that is:

$$
\left|f_{*}^{n}(\rho)^{(s)}\right| \leq T \sum_{i=0}^{s} \sum_{j=0}^{n}\left(\left.f^{n-j}\right|_{E_{n-j}}\right)_{(i)}\left(\tilde{\rho}_{j}^{(i)}\right),
$$

where $T$ is related to the map $\left.f^{n-j}\right|_{E_{n-j}}$ and hence it is uniformly bounded on $I$. This makes it necessary to generalize (3) to $\left(\left.f^{k}\right|_{E_{k}}\right)_{(i)}$, which is proved in lemma 5.1 and prepared in $\S 2$, and to generalize (2) to $\left(\left(\left.f^{j}\right|_{U_{j}}\right)_{*}(\rho)\right)^{(i)}$, which is done in lemma 5.2. To get the generalized (2), we also have to generalize (4) to $\left(f_{*}^{n}(\rho)\right)^{(s)}$. This is done in $\S 4$. It follows from (6), (5) and a formula similar to (5) for $f_{(i)}^{n}\left(\varphi_{i}\right)$ which is proved in lemma 3.4 .

Now we put the generalized (2) and (3) into (7), and we can see that in order to obtain $\tau<1$ in proposition 5.4 we need to introduce small $\varepsilon>0$ in the definition of $\varphi_{i}((3.10))$.

\section{Derivative lemmas}

In this section we shall consider maps $f: I \backslash V \rightarrow I, r$-times differentiable $(r \geq 2)$ and such that $f^{\prime} \neq 0$, for $V$ a countable sum of intervals. In what follows $f^{-n}$ denotes a branch of the inverse map for $f^{n}$ and $f^{-k}(k<n)$ is the branch of the inverse map for $f^{k}$ related to $f^{-n}$, i.e. whenever $f^{-n}=S_{n} \circ \cdots \circ S_{1}$ then $f^{-k}=S_{k} \circ \cdots \circ S_{1}$, where $S_{j}$ $(j=1, \ldots, n)$ is the inverse for $\left.f\right|_{J}, J$ a component of $I \backslash V$. The compositions of functions are restricted to the sets where they are well defined. Next, $\rho: I \rightarrow \mathbb{R}$ is a real function $(r-1)$-times differentiable on some subset of $\hat{I}$.

LEMMA 1.1. Let $x \in \hat{I}$ be such that $y=f^{-n}(x),\left(f^{n}\right)^{(r)}(y)$ and $\rho^{(r-1)}(y)$ are well defined and $\left(f^{n}\right)^{\prime}(y) \neq 0$. Then for $s=0, \ldots, r-1$,

$$
\frac{d^{s}}{d x^{s}}\left(\frac{\rho}{\left|\left(f^{n}\right)^{\prime}\right|} \circ f^{-n}\right)(x)
$$

is equal to the sum (modulo signs) of terms like

$$
S\left(l_{0}, Z\right)(x)=D^{l_{0}}(x) \prod_{(l, k, j) \in Z} D_{k}^{l}(x)^{j},
$$

where $Z$ is a set of triples of integers $(l, k, j)(1 \leq l \leq s ; 1 \leq k \leq n$ and for each pair $(l, k)$ there is only one $j$ such that $(l, k, j) \in Z)$ such that $l_{0}+\sum\{l \cdot j:(l, k, j) \in Z$ for certain $j, k \in \mathbb{N}\}=s, 0 \leq l_{0} \leq s$ and

$$
D_{k}^{l}=\frac{f^{(l+1)}}{\left(\left(f^{k}\right)^{\prime}\right)^{l} f^{\prime}} \circ f^{-k} \quad \text { and } \quad D^{l}=\frac{\rho^{(l)}}{\left(\left(f^{n}\right)^{\prime}\right)^{l+1}} \circ f^{-n} .
$$

Also each term occurs in the sum less than $((s+1) !)^{3}$ times and for $l_{0}=s$ there is only one term $S(s, \varnothing)=D^{s}$.

Proof. We omit elementary justification of formulae (1.1), (1.2). First let us find the derivatives of all components of one term:

$$
\begin{aligned}
& \frac{d}{d x} D^{l}=D^{l+1}-(l+1) \cdot D^{l} \cdot \sum_{k=1}^{n} D_{k}^{l} \\
& \frac{d}{d x} D_{k}^{l}=D_{k}^{l+1}-D_{k}^{l}\left(l \sum_{i=1}^{k} D_{i}^{1}+D_{k}^{l}\right) .
\end{aligned}
$$


This enables us to find the derivative of such a term:

$$
\begin{aligned}
\frac{d}{d x} S\left(l_{0}, Z\right)= & S\left(l_{0}+1, Z\right)-\left(l_{0}+1\right) \sum_{k=1}^{n} S\left(l_{0}, Z\right) \cdot D_{k}^{1} \\
& +\sum_{(l, k, j) \in Z} j \cdot\left[S\left(l_{0}, Z\right) \frac{D_{k}^{l+1}}{D_{k}^{l}}-l \cdot \sum_{i=1}^{k} S\left(l_{0}, Z\right) \cdot D_{i}^{1}-S\left(l_{0}, Z\right) \cdot D_{k}^{1}\right] .
\end{aligned}
$$

Now we can prove the first part of the lemma using induction. Formula (1.1) for $l=0$ proves the lemma when $s=1$. Induction and formula $(1.3)$ then proves the lemma for all $s \leq r-1$.

We now proceed to estimate $N(s)$, the maximal number of repetitions of one term of the sth derivative. Let us fix the term $S=S\left(l_{0}, Z\right)$, which occurs in the $s+1$ st derivative and list all the ways it could originate. We make use of the formula (1.3).

(I) $S$ could originate from $S_{1}=S / D_{k}^{1}$ (if $S$ contains the component $D_{k}^{1}$ ). The number of such $S_{1}$ is less than or equal to $s-l_{0}$. Taking the derivative of $S_{1}$ we can obtain at most $\left(l_{0}+1\right)+\left(s-l_{0}+1\right)^{2}$ terms $S$. Hence one can obtain at most $\left(\left(l_{0}+1\right)+\right.$ $\left.\left(s-l_{0}+1\right)^{2}\right)\left(s-l_{0}\right)$ terms $S$ this way.

(II) One $S$ appears from $S\left(l_{0}-1, Z\right)$.

(III) $S$ might originate from $S_{2}=S \cdot D_{k}^{l-1} / D_{k}^{l}$ (if $S$ contains the component $\left.D_{k}^{l}, l \geq 2\right)$. There are at most $\left(s-l_{0}\right) / 2$ such $S_{2}$. Differentiating $S_{2}$ we obtain at most $s-l_{0}$ terms $S$. Hence no more than $\left(s-l_{0}\right)^{2} / 2$ terms $S$ originate this way.

From (I)-(III) we have (omitting some details) that the number of terms $S$ is less than or equal to:

$$
\left\{\left(\left(l_{0}+1\right)+\left(s-l_{0}+1\right)^{2}\right)\left(s-l_{0}\right)+\left(\left(s-l_{0}\right)^{2} / 2\right)+1\right\} N(s) \leq s(s+1)(s+2) N(s) .
$$

Notice that in view of $(1.1), N(1)=1$. Hence

$$
N(s) \leq(s-1) ! s !(s+1) ! \leq((s+1) !)^{3} \text {. }
$$

The last statement of the lemma follows easily from (1.1) and induction on $s$.

Denote by $\mathscr{L}\left(l_{0}, s, f^{-n}\right)$ the set of all $Z$ such that $S\left(l_{0}, Z\right)$ occurs in the formula which expresses the $s$ th derivative in lemma 1.1. Next define the following functions $T(i, s, n): I \rightarrow \mathbb{R}^{+}$.

$$
\begin{aligned}
& T(i, s, n)=\max \left\{R\left(i, s, f^{-n}\right): f^{-n} \text { is a branch of the inverse for } f^{n}\right\} \\
& T(s, s, n) \equiv 1, \quad(i, s, n \in \mathbb{N}, i<s<r)
\end{aligned}
$$

where for $i<s$

$$
R\left(i, s, f^{-n}\right)(x)= \begin{cases}\sum_{Z \in \mathscr{X}\left(i, s, f^{-n}\right)} \prod_{(l, k, j) \in Z}\left|D_{k}^{l}(x)\right|^{j} & \text { if } x \text { belongs to the domain of } f^{-n} \\ 0 & \text { elsewhere on } I .\end{cases}
$$

Let us define the operators $f_{(i)}(i=0,1,2, \ldots)$ :

$$
f_{(i)}(\rho)(x)=\sum_{y \in f^{-1}(x)} \frac{\rho(y)}{\left|f^{\prime}(y)\right|^{i+1}} \quad \text { for } \rho: I \rightarrow \mathbb{R} .
$$


We remark that

$$
f_{(i)} \circ g_{(i)}=(f \circ g)_{(i)}
$$

and hence $\left(f^{n}\right)_{(i)}=\left(f_{(i)}\right)^{n}$. Therefore we shall often write $f_{(i)}^{n}$ instead of $\left(f^{n}\right)_{(i)}$ or $\left(f_{(i)}\right)^{n}$.

Notice that $f_{(0)}$ is the Perron-Frobenius operator. We shall also denote it by $f_{*}$. LEMMA 1.2. Let $x \in \hat{I}$ be such that for each $y$ with $f^{n}(y)=x,\left(f^{n}\right)^{(r)}(y)$ and $\rho^{(r-1)}(y)$ are well defined and $\left(f^{n}\right)^{\prime}(y) \neq 0$. Then for $s \leq r-1$

$$
\left|f_{*}^{n}(\rho)^{(s)}(x)\right| \leq \sum_{i=0}^{s} f_{(i)}^{n}\left(\left|\rho^{(i)}\right|\right)(x) T(i, s, n)(x) .
$$

Proof. For a fixed branch $f^{-n}$, from lemma 1.1 it follows that

Consequently

$$
\begin{aligned}
\left|\frac{d^{s}}{d x^{s}}\left(\frac{\rho}{\left|\left(f^{n}\right)^{\prime}\right|} \circ f^{-n}\right)(x)\right| & \leq \sum_{l_{0}=0}^{s-1}\left|D^{l_{0}}(x)\right| \cdot R\left(l_{0}, s, f^{-n}\right)(x)+\left|D^{s}(x)\right| \\
& \leq \sum_{l_{0}=0}^{s} T\left(l_{0}, s, n\right)(x) \cdot\left|D^{l_{0}}(x)\right| .
\end{aligned}
$$

$$
\begin{aligned}
\left|f_{*}^{n}(\rho)^{(s)}(x)\right| & \leq \sum_{f^{-n}}\left|\left(\frac{\rho}{\left|\left(f^{n}\right)^{\prime}\right|^{l_{0}+1}} \circ f^{-n}\right)^{(s)}(x)\right| \\
& \leq \sum_{l_{0}=0}^{s} T\left(l_{0}, s, n\right)(x) \sum_{f^{\prime n}}\left|D^{l_{0}}(x)\right| \\
& =\sum_{l_{0}=0}^{s} T\left(l_{0}, s, n\right)(x) f_{\left(l_{0}\right)}^{n}\left(\mid \rho^{\left(l_{0}\right)}\right)(x) .
\end{aligned}
$$

This completes the proof.

Remark 1.3. Let $E$ be a sum of pairwise disjoint intervals. Then lemma 1.2 remains true if one replaces $f^{n}$ by $\left.f^{n}\right|_{E}$ and the branches $f^{-n}$ by respective branches of the inverse for $\left.f^{n}\right|_{E}$. (They are certain restrictions of branches $f^{-n}$.)

2. Expansion in the class $\mathcal{M}$

I shall list the conditions found by $M$. Misiurewicz [6] in a slightly stronger version. They are satisfied for an uncountable subset of the family $\{\alpha x(1-x)\}$, in particular when some image of the critical point falls into the repelling periodic orbit.

(i) $f$ is of class $C^{3}$.

(ii) $f^{\prime} \neq 0$.

(iii) $S f \leq 0$, and for each $a \in \hat{A_{1}}$ there exists $\delta>0$ and a function $g:[\check{a}-\delta, \check{a}+\delta] \rightarrow$ $\mathbb{R}$ such that $g$ is of class $C^{3}, g^{\prime} \neq 0, S g \leq 0$ and $\left.g\right|_{F(a, \delta)}=\left.f\right|_{F(a, \delta)}$.

(iv) For each $x \in \hat{I}$, if $f^{p}(x)=x$ then $\left|\left(f^{p}\right)^{\prime}(x)\right|>1$.

(v) There exists a neighbourhood $\mathcal{U}$ of $A$, such that for each $a \in \hat{A}$ and $n \geq 0$, $\left(f^{n}(a)\right)^{\vee} \in I \backslash \mathcal{U} \cup A$.

Also for each $a \in A$ not being the end of $I,(a,+)$ or $(a,-)$ belongs to $\hat{A}_{2}$.

(vi) For each $a \in \hat{A}$ there exist constants $\alpha, \omega, \delta>0$ and $u \geq 0$ such that

$$
\alpha|x-\check{a}|^{u} \leq\left|f^{\prime}(x)\right| \leq \omega|x-\check{a}|^{u}
$$

for every $x \in U_{a}=F(a, \delta)$.

We define $\mathcal{M}$ as the class of maps $f$ satisfying conditions (i)-(vi). 
Conditions (iii) and (v) are a little stronger than the respective ones formulated by $M$. Misiurewicz [6]. The second part of (iii) is introduced to obtain a more explicit form of weight functions (see 3.10). The second part of (v) together with (iii) enables us to obtain the very useful theorem 2.1 as an easy consequence of theorem (1.3) in [6]. We remark that (iv) implies non-existence of sinks and that (vi) is satisfied whenever $f$ has non-zero one-sided derivatives of some order at points of $A$.

Let $f$ satisfy (i)-(iv) and the second part of (v). Let us define for $\gamma>0$ :

$$
W_{\gamma}=\bigcup_{a \in \hat{A}_{2}} F(a, \gamma) \cup\left(A \backslash\left(\hat{A}_{1}\right)^{\vee}\right) \text {. }
$$

THEOREM 2.1. For each $\delta>0$ there exists $m \in \mathbb{N}$ such that if $f^{i}(x) \in I \backslash W_{\delta}$ for $i=0, \ldots, m-1$ then $\left|\left(f^{m}\right)^{\prime}(x)\right|>1$.

Proof. Since $f$ satisfies (iii) and the second part of (v), $\left.f\right|_{I \backslash W_{8}}$ can be extended to a function $h: V \rightarrow I$ where $V$ is a neighbourhood of $I \backslash W_{\delta}$ (we might have to increase $I$ ). Such an $h$ satisfies the assumptions of theorem (1.3) of [6], and hence theorem 2.1 follows.

LEMMA 2.2(a) (Distortion lemma). For each $\delta>0$, there exists $\rho>0$ such that if $f^{j}(x)$ and $f^{j}(y)$ belong to the same component of $I \backslash W_{\delta}$ for $j=0, \ldots, n-1$ then

$$
\left|\frac{\left(f^{n}\right)^{\prime}(x)}{\left(f^{n}\right)^{\prime}(y)}\right| \leq \rho \text {. }
$$

(b) Let $f$ satisfy (vi). For each $\delta>0$ there exists $\sigma>0$ such that if $x \in U_{a}$ and $f^{j}(x),\left(f^{j}(a)^{\vee}\right.$ belong to the same component of $I \backslash W_{\delta}$ for $j=1,2, \ldots, n-1$ then

$$
\frac{1}{\sigma} \leq(u(a)+1)\left|\frac{\left(f^{n}\right)^{\prime}(x)(x-\check{a})}{f^{n}(x)-\left(f^{n}(a)\right)^{v}}\right| \leq \sigma .
$$

Proof. (a) Let $K$ be a component of the set $I \backslash \bigcup_{i=0}^{n-1} f^{-j}\left(W_{\delta}\right)$ and $x, y \in K$. Following (1.1) of [6] we have:

$$
\left|\frac{\left(f^{n}\right)^{\prime}(x)}{\left(f^{n}\right)^{\prime}(y)}\right| \leq \exp \left(\gamma \sum_{k=0}^{n-1} \lambda\left(f^{k}(K)\right)\right),
$$

where $\gamma>0$ is a Lipshitz constant of $\ln \left|h^{\prime}\right|$ (see the proof of theorem 2.1) and $\lambda$ is Lebesgue measure.

Theorem 2.1 gives us that the derivative on $K$ grows exponentially with $n$ (of course, $K$ also changes with $n)$. Namely, $\left|\left(f^{n}\right)^{\prime}(x)\right|>A^{\prime} \bar{\alpha}^{n}$ for certain constants $A^{\prime}>0$ and $\bar{\alpha}>1$ and $x \in K$. Hence

and consequently

$$
\lambda(I) \geq \lambda\left(f^{n}(K)\right) \geq A^{\prime} \cdot \bar{\alpha}^{n-k} \cdot \lambda\left(f^{k}(K)\right)
$$

$$
\sum_{k=0}^{n-1} \lambda\left(f^{k}(K)\right) \leq \lambda(I) / A^{\prime} \sum_{k=0}^{n-1} \bar{\alpha}^{k-n} \leq \frac{\bar{\alpha} \cdot \lambda(I)}{(\bar{\alpha}-1) A^{\prime}} .
$$

Thus (a) follows for $\rho=\exp \left(\gamma \bar{\alpha} \cdot \lambda(I) /(\bar{\alpha}-1) \cdot A^{\prime}\right)$.

(b) From (vi) we obtain

$$
\frac{\alpha}{u+1}|x-\check{a}|^{u+1} \leq\left|f(x)-(f(a))^{v}\right| \leq \frac{\omega}{u+1}|x-\check{a}|^{u+1},
$$


so we have

$$
\begin{aligned}
\left|f^{n}(x)-\left(f^{n}(a)\right)^{v}\right| & =\left|f^{n-1}(f(x))-f^{n-1}(f(a))^{v}\right| \\
& =\left|\left(f^{n-1}\right)^{\prime}(\xi)\right| \cdot\left|f(x)-(f(a))^{v}\right| \\
& =\left|\frac{\left(f^{n-1}\right)^{\prime}(\xi)}{\left(f^{n-1}\right)^{\prime}(f(x))}\right| \cdot \frac{\left|f(x)-(f(a))^{v}\right|}{\left|f^{\prime}(x)\right|} \cdot\left|\left(f^{n}\right)^{\prime}(x)\right|
\end{aligned}
$$

where $\xi$ is some number between $f(x)$ and $(f(a))^{\vee}$. Then $f^{j}(\xi)$ is between $f^{j+1}(x)$ and $\left(f^{j+1}(a)\right)^{\vee}$ and they all belong to the same component of $I \backslash W_{\delta}$. Hence by the distortion lemma, (vi), (2.1) and (2.2):

$$
\begin{aligned}
\frac{\alpha}{\rho \cdot \omega \cdot(u+1)}|x-\check{a}| \cdot\left|\left(f^{n}\right)^{\prime}(x)\right| & \leq\left|f^{n}(x)-\left(f^{n}(a)\right)^{v}\right| \\
& \leq \frac{\rho \omega}{\alpha(u+1)}|x-\check{a}| \cdot\left|\left(f^{n}\right)^{\prime}(x)\right| .
\end{aligned}
$$

This proves (b) with $\sigma=\max _{a \in \hat{A}_{2}} \rho \cdot \omega(a) / \alpha(a)$.

The following lemma was proved by K. Ziemian [11] in a slightly different form.

LEMMA 2.3. Let $f$ belong to $\mathcal{M}$.

(a) If $x \in U_{a}$ for some $a \in \hat{A}$ and $\gamma>0$ is sufficiently small, then

$$
n(x, \gamma)=\min \left\{i \in \mathbb{N}:\left|f^{i}(x)-\left(f^{i}(a)\right)^{\vee}\right| \geq \gamma\right\}
$$

is well defined.

(b) For each $\gamma>0$ there exists a constant $A^{\prime}>0$ such that if $x \in U_{a}$, dist $\left(f^{\prime}(x)\right.$, $\left.\hat{B}_{l}\right) \geq \gamma$ and $l \geq n(x, \gamma)$ then

$$
\left|\left(f^{\prime}\right)^{\prime}(x)\right| \geq \frac{\boldsymbol{A}^{\prime}}{|x-\check{a}|} .
$$

(c) For each $\gamma>0$ small enough there exist constants $E>0$ and $c>1$ such that if $\operatorname{dist}\left(f^{l}(x), \hat{B}_{l}\right) \geq \gamma$ then $\left|\left(f^{l}\right)^{\prime}(x)\right|>E c^{l}$.

Lemma 2.3 can be proved from lemma 2.2 .

\section{Estimates far from singularities}

For $b \in \hat{I}$ let us define the function $\psi_{b}: I \rightarrow \mathbb{R}$ :

$$
\psi_{b}(x)= \begin{cases}\frac{1}{|x-\check{b}|} & \text { for } x>\check{b} \text { if } \underline{b}=+, \text { and for } x<\check{b} \text { if } \underline{b}=- \\ 0 & \text { elsewhere. }\end{cases}
$$

LeMMA 3.1. Let $f$ satisfy (i)-(iii) and let us fix some $\eta, 0<\eta<1$. Then for any $x \in I$

$$
\begin{aligned}
f_{*}^{n}\left(\psi_{b}^{\eta}\right)(x) & \leq \frac{\int \psi_{b}^{n} d \lambda}{\operatorname{dist}\left(x, B_{n} \cup\left\{f^{n}(b)\right\}\right)}, \\
f_{*}^{n}(1)(x) & \leq \frac{\lambda(I)}{\operatorname{dist}\left(x, \hat{B}_{n}\right)} .
\end{aligned}
$$

Proof. Let $J$ be a component of $I \backslash\left(\{\check{b}\} \cup \bigcup_{k=0}^{n-1} f^{-k}(A)\right)$, such that $\left.\psi_{b}\right|_{J}>0$. Then $\left.f^{n}\right|_{J}$ is a diffeomorphism. Denote by $(a, \bar{b})$ the interval $f^{n}(J)$ and let $g=\left.f^{n}\right|_{J}$. Notice 
that $(a,+),(\bar{b},-) \in \hat{B}_{n} \cup\left\{f^{n}(b)\right\}$. Let us recall the formula for the Schwarzian derivative of a composition.

$$
S(g \circ h)=\left(h^{\prime}\right)^{2}(S g \circ h)+S h .
$$

Write $\psi_{b}^{\eta}=\rho$.

By lemma 4.1 of $[6]$, since $1 / \sqrt{\left.\rho\right|_{J}}$ is concave and $\left.\rho\right|_{J}$ is $C^{2},\left.\rho\right|_{J}=h_{*}(1)$ for some negative Schwarzian diffeomorphism $h$. In view of formula (3.1) $S(g \circ h) \leq 0$. We see that

$$
g_{*}\left(\left.\rho\right|_{J}\right)=g_{*}\left(h_{*}(1)\right)=(g \circ h)_{*}(1) .
$$

Hence by lemma 4.1 of [6], $1 / \sqrt{g_{*}\left(\left.\rho\right|_{J}\right)}$ is concave. Then $\sqrt{g_{*}\left(\left.\rho\right|_{J}\right)}$ is convex and consequently $g_{*}\left(\left.\rho\right|_{J}\right)$ is convex. Thus

$$
\begin{aligned}
g_{*}\left(\left.\rho\right|_{J}\right)(x) & \leq\left\{\begin{array}{l}
\frac{1}{x-a} \int_{a}^{x} g_{*}\left(\left.\rho\right|_{J}\right) d \lambda=\frac{\int_{I_{1}} \rho d \lambda}{x-a} \\
\frac{1}{\bar{b}-x} \int_{x}^{\bar{b}} g_{*}\left(\left.\rho\right|_{J}\right) d \lambda=\frac{\int_{I_{2}} \rho d \lambda}{\bar{b}-x}
\end{array}\right. \\
& \leq \frac{\int_{J} \rho d \lambda}{\operatorname{dist}\left(x, \hat{B}_{n} \cup\left\{f^{n}(b)\right\}\right)},
\end{aligned}
$$

where $x \in(a, \bar{b}), I_{1}=\mathrm{g}^{-1}((a, x))$ and $I_{2}=\mathrm{g}^{-1}((x, \bar{b}))$. We finish the proof by summing the above inequalities over all components $J$.

A proof of the second inequality is similar.

LEMMA 3.2. Let $f \in \mathcal{M}$. For each $\gamma>0, i \in \mathbb{N}, 0<\xi<1$ and $\xi<\eta<1$, there exist constants $C>0$ and $0<g<1$ such that

$$
f_{(i)}^{n}\left(\psi_{b}^{i+\xi}\right)(x) \leq C g^{n} f_{*}^{n}\left(\psi_{b}^{\eta}\right)(x),
$$

for $b \in \hat{B}, n \in \mathbb{N}, x \in I$ such that $\operatorname{dist}(x, \hat{B}) \geq \gamma$.

Proof. Let us write

$$
\frac{\psi_{b}^{i+\xi}}{\left|\left(f^{n}\right)^{\prime}\right|^{i+1}}=\frac{\psi_{b}^{n}}{\left|\left(f^{n}\right)^{\prime}\right|} \tau^{i}
$$

where $\tau: I \backslash \bigcup_{i=0}^{n-1} f^{-i}(A) \rightarrow \mathbb{R}, \tau(y)=\psi_{b}^{\zeta}(y) /\left|\left(f^{n}\right)^{\prime}(y)\right|$ and $\zeta=(i+\xi-\eta) / i<1$. We shall first prove the following assertion:

(3.4) For $\gamma$ and $\xi$ as above there exist $\varepsilon>0, D>0$ and $0<d<1$ such that for each branch $f^{-n}$ of the inverse of $f^{n}$, if $y=f^{-n}(x) \in F(b, \varepsilon)$ then $\tau(y) \leq D d^{n}$, where $x$ and $b$ are as in the lemma.

Let us fix some $\delta>0$ such that $W_{\delta}$ (see (2.0)) is separated from $B \backslash\left(\hat{A}_{1}\right)^{\vee}$ (see (v) of $\S 2$ ) and contained in $\bigcup_{a \in \hat{A}_{2}} U_{a} \cup A$ (see (vi)). Suppose that $b$ in the lemma is equal to $f^{k}(a)$ for some $a \in \hat{A}_{2}$ and $k \in \mathbb{N}$. We take $\varepsilon$ small enough that $\gamma>\varepsilon>0$ and the following conditions (3.5) and (3.6) are satisfied.

For $m \in \mathbb{N}$, let $f^{-m}$ be the branch of the inverse for $f^{m}$ related to a trajectory of $a$ (i.e. for small $\left.\rho>0,\left(\left.f^{m}\right|_{F(a, \rho)}\right)^{-1}=\left.f^{-m}\right|_{f^{m}(F(a, \rho))}\right)$.

(3.5) $f^{-m}$ is defined on $F=F\left(f^{m}(a), \varepsilon\right)$ and $f^{-m}(F) \subset U_{a} \cap W_{\delta}, m \in \mathbb{N}$. 
Next, for $m \in \mathbb{N}$ and $j=1,2, \ldots, m$, let $f^{-j}$ be the branch of the inverse for $f^{j}$ related to $f^{-m}$ (see the beginning of $\S 1$ ) where $f^{-m}$ is from (3.5). Then

(3.6) $f^{-j}(F) \cap W_{\delta}=\varnothing$ and $f^{-j}(F) \subset F\left(f^{m-j}(a), \gamma\right)$ for $j=1, \ldots, m-1$.

The existence of such an $\varepsilon$ follows from theorem 2.1 combined with (v). Namely, this theorem gives us $m_{0} \in \mathbb{N}$ such that $\left|\left(f^{m_{0}}\right)^{\prime}\right|_{B} \mid>1$ and consequently for some $\varepsilon_{1}>0$ $\left|\left(f^{m_{0}}\right)^{\prime}\right|_{U_{\varepsilon_{1}}} \mid>g>1$, and $U_{\varepsilon_{1}} \cap \bigcup_{i=0}^{m_{0}-1} f^{-i}(A)=\varnothing$, where $U_{\varepsilon_{1}}=\left\{x: \operatorname{dist}(x, \hat{B})<\varepsilon_{1}\right\}$. Hence (3.5), (3.6) for $m=m_{0} \cdot l+1, l \in \mathbb{N}$, follow easily by taking some $\varepsilon_{2} \leq \varepsilon_{1}$ such that $U_{\varepsilon_{2}} \cap W_{\delta}=\varnothing$. This implies (3.5), (3.6) for $m \in \mathbb{N}$ with $\varepsilon \leq \varepsilon_{2}$ such that $f^{-j}\left(F\left(f^{j}(b), \varepsilon\right)\right) \subset F\left(b, \varepsilon_{2}\right)$ for all $b \in \hat{B}, j=1, \ldots, m_{0}-1$ and $f^{-j}$ is as in (3.6). This briefly justifies (3.5), (3.6).

Let us take $l=n(z, \gamma)-k$ (see lemma 2.3a), where $z=f^{-k}(y)$ and $f^{-k}$ is the branch related to a trajectory of $a$. We can do this because of (3.6) for $m=k$. From (3.6) we have that $l \geq 0$ and since $\left|x-\left(f^{n+k}(a)\right)^{\vee}\right| \geq \gamma, l \leq n$. Next, since $y \in F(b, \varepsilon)$, from (3.5), (3.6) and lemma $2.2 \mathrm{~b}$ it follows that

$$
\begin{gathered}
(u+1) \cdot\left|\frac{\left(f^{k}\right)^{\prime}(z) \cdot(z-\check{a})}{y-\check{b}}\right| \leq \sigma, \\
\frac{1}{\sigma} \leq(u+1) \cdot\left|\frac{\left(f^{l+k}\right)^{\prime}(z) \cdot(z-\check{a})}{f^{l}(y)-\left(f^{l}(b)\right)^{\vee}}\right|
\end{gathered}
$$

and consequently we have

$$
\begin{aligned}
|y-\breve{b}| & \geq \frac{u+1}{\sigma}\left|\left(f^{k}\right)^{\prime}(z)\right| \cdot|z-\check{a}| \geq \sigma^{-2} \frac{\left|f^{l}(y)-f^{l}(b)^{v}\right|}{\left|\left(f^{l}\right)^{\prime}(y)\right|} \\
& \geq \frac{\sigma^{-2} \gamma}{\left|\left(f^{l}\right)^{\prime}(y)\right|} .
\end{aligned}
$$

(Notice that $\left(f^{l+k}\right)^{\prime}(z)=\left(f^{l}\right)^{\prime}(y) \cdot\left(f^{k}\right)^{\prime}(z)$.) We proceed to estimate $\tau$.

From (3.7) and lemma $2.3 \mathrm{c}$ we have:

where

$$
\tau(y) \leq \frac{\left(\sigma^{2} \gamma^{-1}\right)^{\zeta}}{\left|\left(f^{n-1}\right)^{\prime}\left(f^{l}(y)\right)\right| \cdot\left|\left(f^{l}\right)^{\prime}(y)\right|^{1-\zeta}} \leq D d^{n},
$$

$$
D=\frac{\left(\sigma^{2} \gamma^{-1}\right)^{\zeta}}{E^{2-\zeta}} \text { and } d=\left(\frac{1}{c}\right)^{1-\zeta}<1 .
$$

(Notice that $\left(f^{n}\right)^{\prime}(y)=\left(f^{n-l}\right)^{\prime}\left(f^{l}(y)\right) \cdot\left(f^{l}\right)^{\prime}(y)$ and $0<\zeta<1$.) This completes the proof of (3.4).

(3.9) There exist constants $H>0$ and $0<h<1$ such that $\tau(y) \leq H h^{n}(n \in \mathbb{N})$ for $y \in I$ such that $f^{n}(y)=x$ and dist $(x, \hat{B})>\gamma$.

In order to see this, one has to consider the case $\operatorname{dist}(y, \hat{B})>\varepsilon$ together with the proof of (3.4). By lemma $2.3 \mathrm{c}$ it is very easy.

Now from (3.3), (3.9) and the definition of $f_{(i)}^{n}$ it follows that

$$
f_{(i)}^{n}\left(\psi_{b}^{i+\xi}\right)(x)=\sum_{y \in f^{-n}(x)} \frac{\psi_{b}^{\eta}(y)}{\left|\left(f^{n}\right)^{\prime}(y)\right|}(\tau(y))^{i} \leq H^{i}\left(h^{i}\right)^{n} \cdot f_{*}^{n}\left(\psi_{b}^{\eta}\right)
$$

and this proves the lemma for $C=D^{i}$ and $g=d^{i}$. 
We proceed to define the weight functions $\varphi_{i}$ which will enable us to construct Banach spaces $C_{f, \varepsilon}^{r-1}$ invariant under the Perron-Frobenius operator. For $f \in \mathcal{M}$ and $i \in \mathbb{N}$ or $i=\varepsilon \in\left[0, \min _{a \in \hat{A}_{2}}(1-\xi(a)) / 2\left[\right.\right.$ we define $\varphi_{i}: I \backslash B_{0} \rightarrow \mathbb{R}^{+}:$

$$
\varphi_{i}(x)=1+\sum_{n=0}^{+\infty} \sum_{a \in \hat{A}_{2}}\left|\left(f^{n}\right)^{\prime}\left(a_{1}\right)\right|^{-\eta(a)} \psi_{a_{n+1}}^{i+\xi(a)}(x),
$$

where $\eta(a)=1-\xi(a)-2 \varepsilon, \quad \xi(a)=u(a) /(u(a)+1), \quad a_{j}=f^{j}(a)$ for $j \in \mathbb{N}, B_{0}=$ $\bigcap_{\gamma>0} U_{\gamma}$ and $U_{\gamma}=\bigcup_{b \in \hat{B}} F(b, \gamma)$.

Since for some $x<+\infty, \sup \left|f^{\prime}\right| \leq x, f\left(U_{\gamma}\right) \subset U_{x y}$ and hence

$$
f\left(B_{0}\right) \subset B_{0} \text {. }
$$

In what follows if $\rho: I \backslash B_{0} \rightarrow \mathbb{R}$ then we regard $f_{*}(\rho): I \backslash B_{0} \rightarrow \mathbb{R}$. Since $f^{-1}\left(I \backslash B_{0}\right) \subset I \backslash B_{0}$ (see (3.11)), $f_{*}(\rho)$ is well defined.

We remark also that since $\left|\left(f^{n}\right)^{\prime}\left(a_{1}\right)\right|$ grows exponentially (see (v) and theorem $2.1)$ and $\hat{A}_{2}$ is finite and the functions $\left\{\psi_{a_{n+1}+\xi(a)}\right\}_{n \in N}$ have uniformly bounded integrals, $\varphi_{\varepsilon} \in L_{1}(\lambda)$. Similarly from the definition of $B_{0}$ it follows that

$$
\varphi_{i}(x)<+\infty \quad \text { for } x \in I \backslash B_{0}, i \in \mathbb{N} \text { or } i=\varepsilon .
$$

Proposition 3.3. Let $x \in I \backslash B_{0}$. Then

$$
\operatorname{dist}(x, \hat{B})>0 \text { and } f_{*}^{n}\left(\varphi_{\varepsilon}\right)(x) \leq \frac{\int \varphi_{\varepsilon} d \lambda}{\operatorname{dist}(x, \hat{B})}
$$

Proof. Suppose $x \notin B_{0}$. Then $x \notin U_{\gamma}$ for some $\gamma>0$ and hence the first inequality follows. One obtains the second inequality by adding the inequalities obtained from lemma 3.1 applied to all the functions $\left\{\psi_{a_{m+1}}^{E+\xi(a)}\right\}_{m \in N, a \in \hat{A}_{2}}$ together with the function 1.

Lemma 3.4. Let $f \in \mathcal{M}$. For each $\gamma>0$ and $i \in \mathbb{N}$ there exist constants $D>0$ and $0<c<1$ such that

$$
f_{(i)}^{n}\left(\varphi_{i}\right)(x) \leq D c^{n}
$$

for $n \in \mathbb{N}$ and for $x \in I$ such that $\operatorname{dist}(x, \hat{B}) \geq \gamma$.

Proof. We remark first that by lemma $2.3 \mathrm{c}$ we can find constants $C>0$ and $0<\mathrm{g}<1$ such that

$$
f_{(i)}^{n}(1)(x) \leq C g^{n} f_{*}^{n}(1)(x),
$$

for $n \in \mathbb{N}$ and $x$ as above. Thus from lemma 3.2 and (3.10), for each small $\varepsilon>0$ we have (adding the inequalities in turn) $C>0$ and $0<g<1$ such that

$$
f_{(i)}^{n}\left(\varphi_{i}\right)(x) \leq C g^{n} f_{*}^{n}\left(\varphi_{\varepsilon}\right)
$$

Using proposition 3.3, the proof is completed by putting $D=C \int \varphi_{\varepsilon} d \lambda / \gamma$ and $c=g$.

4. The class $\mathcal{M}^{r}$ and Banach spaces $C_{f, \varepsilon}^{r-1}$

We introduce a new condition:

(vi') $f$ can be extended to be of class $C^{r}, r \in \mathbb{N}$, on $I \backslash W_{\gamma}$ (see (2.0)) for all $\gamma>0$, and for each $s, 1<s \leq r$ and $a \in \hat{A}_{2}$ there exist constants $\kappa>0$ and $v=v(s, a) \geq$ $u(a)-s+1$ such that $\left|f^{(s)}(x)\right| \leq \kappa|x-a ̆|^{v}$ for $x \in U_{a}$ (see (vi)). 
We remark that $\left(\mathrm{vi}^{\prime}\right)$ is valid whenever $f$ has non-zero one-sided derivatives of some order at $a \in A$.

In what follows $\mathcal{M}^{r}$ will be the class of maps satisfying (i)-(vi') (see $\$ 2$ and notice that $\mathcal{M}^{r} \subset \mathcal{M}$ ).

We are going to estimate the derivatives of the iterates $f_{*}^{n}(\rho)$ far from their singularities. Let us recall that $U_{\gamma}=\bigcup_{b \in \hat{B}} F(b, \gamma)$.

LEMMA 4.1. Let $f \in \mathcal{M}^{r}$. For each $\gamma>0$ small enough there exist constants $B>0, c<1$ such that for each branch of $f^{-n}$ if $x \notin U_{\gamma}$ then

$$
\left|D_{k}^{\prime}(x)\right| \leq\left(B / c^{k^{\prime}}\right)^{l}
$$

where $0 \leq l<r, k=1,2, \ldots, n$ and

$$
k^{\prime}= \begin{cases}k & \text { if } f^{-k}(x) \notin W, \\ \max \left(\left\{i: 1 \leq i<k, f^{-i}(x) \in W\right\} \cup\{0\}\right) & \text { if } f^{-k}(x) \in W .\end{cases}
$$

( $D_{k}^{l}$ is from lemma $1.1, W=W_{\delta}$ for some small $\delta$, see (2.0).)

Proof. Let us suppose that $f^{-k}(x) \notin W$. Then by (vi'), $\sup _{y \ll W}\left|f^{(l+1)}(y)\right|=a(l)<+\infty$ and $\inf _{y \in w}\left|f^{\prime}(y)\right|=b>0$. Hence

and by lemma $2.3 \mathrm{c}$

$$
\left|D_{k}^{\prime}(x)\right| \leq \frac{a(l)}{\left|\left(f^{k}\right)^{\prime}\right|^{l} \cdot b} \circ f^{-k}(x)
$$

$$
\left|D_{k}^{l}(x)\right| \leq\left(B(l) / c^{k}\right)^{l}
$$

where $B(l)=1 / E(a(l) / b)^{1 / l}$.

Suppose now that $y=f^{-k}(x) \in F(a, \delta)$ and $\gamma$ is small such that $W \cap U_{\gamma}=\varnothing$ (by (v) this is possible), then by lemma $2.3(\mathrm{~b}),(\mathrm{c})$

$$
\left|\left(f^{k}\right)^{\prime}(y)\right|=\left|\left(f^{k}\right)^{\prime}\left(f^{-k^{\prime}}(x)\right)\right| \cdot\left|\left(f^{k-k^{\prime}}\right)^{\prime}(y)\right| \geq E c^{k^{\prime}} \cdot \frac{A^{\prime}}{|y-\check{a}|} .
$$

Since $|y-\check{a}|<\delta$ this, combined with (vi)-(vi') gives

$$
\left|D_{k}^{\prime}(x)\right| \leq\left(\frac{B^{\prime}(l, a)}{c^{k^{\prime}}}\right)^{\prime}
$$

where

$$
B(l, a)=\frac{1}{E \cdot A^{\prime}}\left(\frac{\delta^{v(l+1)-u+l} \cdot \kappa}{\alpha(a)}\right)^{1 / l} .
$$

(We notice that in view of $\left(\mathrm{vi}^{\prime}\right) v(l+1)-u+l \geq 0$.) We finish the proof by setting

$$
B=\max \left(\{B(l): l=1, \ldots, r-1\} \cup\left\{B^{\prime}(l, a): l=1, \ldots, r-1, a \in \hat{A}\right\}\right) .
$$

LEMMA 4.2. Let $f \in \mathcal{M}^{r}$. For each $\gamma>0$ small enough, there exists a constant $C(\gamma)>0$ such that

$$
T(i, s, n)(x) \leq C(\gamma) \quad \text { for } x \in I \backslash U_{r}, n \in \mathbb{N}, 1 \leq i \leq s<r .
$$

Proof. Throughout the proof we fix a branch of $f^{-n}, \gamma>0, x \in I \backslash U_{y}, s \leq r-1$ and $i<s$. We need the following notation: for $Z \in \mathscr{Z}\left(i, s, f^{-n}\right)$,

$$
K(Z)=\{k:(l, k, j) \in Z \text { for some } l, j \in \mathbb{N}\}
$$

and

$$
l(k, Z)=\sum\{l \cdot j:(l, k, j) \in Z\} .
$$


We notice that by lemma $1.1 \sum_{k \in K(Z)} l(k, Z)=s-i$. Let us put $S(Z)=\prod_{(l, k, j) \in Z}\left(D_{k}^{l}\right)^{j}$. From lemma 4.1 it follows that

$$
|S(Z)| \leq \prod_{k \in K(Z)}\left(B / c^{k^{\prime}}\right)^{l(k, Z)} .
$$

Let us estimate $n(Z)=\operatorname{card}\left\{Z_{1} \in \mathscr{Z}\left(i, s, f^{-n}\right): K\left(Z_{1}\right)=K(Z)\right.$ and $l\left(k, Z_{1}\right)=l(k, Z)$ for $k \in K(Z)\}$, the number of different $S\left(Z_{1}\right)$ which have the same estimate (4.1). Since card $\left\{\left(l_{1}, \ldots, l_{t}\right): \sum_{j=1}^{t} l_{j}=m, l_{j}\right.$ positive $\}=2^{m-1}$,

$$
n(Z)<\prod_{k \in K(Z)} 2^{l(k, Z)}=k(s-i),
$$

where

$$
k(m)=2^{m}<+\infty .
$$

(4.1), (4.2) and lemma 1.1 imply

$$
R\left(i, s, f^{-n}\right) \leq((s+1) !)^{3} \cdot k(s-i) \cdot \sum_{\substack{l_{1}+\cdots+l_{l}=s-i \\ 1 \leq k_{1}<\cdots<k_{t} \leq n}} \prod_{j=1}\left(\frac{B}{c^{k_{j}}}\right)^{l_{j}} .
$$

Hence, by Newton's formula

$$
\begin{aligned}
R\left(i, s, f^{-n}\right) & \leq((s+1) !)^{3} k(s-i)\left(\sum_{k=1}^{n} B / c^{k^{\prime}}\right)^{s-i} \\
& \leq((s+1) !)^{3} k(s-i)(B c /(c-1))^{s-i}
\end{aligned}
$$

This completes the proof.

We proceed to define spaces $C_{f, \varepsilon}^{r-1}$ and $C_{f, \varepsilon}^{(r-2)+1}$. Let $x \in I \backslash B_{0}$ and let $J(x, \gamma)$ be the component of $I \backslash U_{\gamma}$ containing $x$. Notice that if $\gamma_{1}<\gamma_{2}$, then $J\left(x, \gamma_{1}\right) \supset J\left(x, \gamma_{2}\right)$ and for $\gamma$ small enough $J(x, \gamma)$ is non-empty. Thus we can define the interval $J(x)=\cup_{\gamma>0} J(x, \gamma)$. Notice that for $x_{1} \neq x_{2}$, either $J\left(x_{1}\right)=J\left(x_{2}\right)$ or $J\left(x_{1}\right) \cap J\left(x_{2}\right)=$ $\varnothing$. Hence the sets $J(x)$ form a countable partition $\Phi$ of $I \backslash B_{0}$. Notice that for $J \in \mathscr{J},\left.\varphi_{i}\right|_{,}, i \in \mathbb{N} \cup\{\varepsilon\}$, is continuous.

For every $\rho: I \backslash B_{0} \rightarrow \mathbb{R}$ we define

$$
\|\rho\|_{\varepsilon}=\sup _{J \in \mathscr{g}} \sup _{J} \frac{|\rho|}{\varphi_{\varepsilon}} .
$$

We suppose now that $\rho$ is of class $C^{s}, s \in \mathbb{N} \cup\{0\}$, on all $J \in \mathscr{F}$. (It may happen that $J=\{x\}$. Then we put $\left(\left.\rho\right|_{J}\right)^{(j)}(x)=0$ for $j \in \mathbb{N}$.) We define for $1 \leq i \leq s$

$$
\begin{aligned}
|\rho|_{i} & =\sup _{J \in \mathscr{I}} \sup _{J} \frac{\left|\rho^{(i)}\right|}{\varphi_{i}}, \\
|\rho|_{1, s} & =\max \left\{|\rho|_{i}: i=1, \ldots, s\right\} .
\end{aligned}
$$

Let us assume in addition that $\rho^{(s)}$ is Lipshitz on compact subsets of all $J \in \mathscr{J}$. Then $\rho^{(s)}$ is almost everywhere differentiable and the following definition makes sense:

$$
|\rho|_{(s)+1}=\sup _{J \in \mathscr{I}} \operatorname{essup} \frac{\left|\rho^{(s+1)}\right|}{\varphi_{s+1}},
$$


and for $s>0$

$$
|\rho|_{1,(s)+1}=\max \left\{|\rho|_{1, s,},\left.\rho\right|_{(s)+1}\right\} .
$$

Finally, if $\rho$ is of class $C^{s}$ on all $J \in \mathscr{J}$ then we define

$$
\|\rho\|_{s}=\max \left\{\|\rho\|_{\varepsilon},|\rho|_{1, s}\right\} \text {, }
$$

and if additionally $\rho$ is as in (4.5) then for $s>0$

$$
\|\rho\|_{(s)+1}=\max \left\{\|\rho\|_{\varepsilon},|\rho|_{1,(s)^{\prime+1}}\right\}
$$

and

$$
\|\rho\|_{(0)+1}=\max \left\{\|\rho\|_{E},|\rho|_{(0)+1}\right\} .
$$

Having these definitions, we can define for $f \in \mathcal{M}$ and $s>0(s=0)$ the space $C_{f, \varepsilon}^{s}$ of all $\rho: I \backslash B_{0} \rightarrow \mathbb{R}$ of class $C^{s}$ on all $J \in \mathscr{J}$ and such that $\|\rho\|_{s}<+\infty\left(\|\rho\|_{\varepsilon}<+\infty\right)$. We define $C_{f, \varepsilon}^{(s)+1}$ to be the space of all $\rho \in C_{f, \varepsilon}^{s}$ such that $\rho^{(s)}$ is Lipshitz on compact subsets of all $J \in \mathscr{J}$ and $\|\rho\|_{(s)+1}<+\infty$.

Remark 4.3(a) If $f \in M^{r}$ and $\rho$ is of class $C^{s}(s \leq r-2)$ and $\rho^{(s)}$ is Lipshitz on compact subsets of $J \in \mathscr{J}$ then $f_{*}(\rho)$ has the same properties.

(b) $C_{f, \varepsilon}^{s}$ and $C_{f, \varepsilon}^{(s)+1}, s \in \mathbb{N} \cup\{0\}$, are Banach spaces.

(c) A ball in $C_{f, \varepsilon}^{(0)+1}$ is compact in $L_{1}(\lambda)$.

Proof. (a) follows easily from the definition of $f_{*}$ and $\mathscr{I}$, (elements of $\mathscr{F}$ are mapped onto elements of $\mathscr{J}$ ).

(b) The proof is based on the fact that a Lipshitz function is a.e. differentiable and is equal to the integral of its derivative. Hence the essential supremum of the derivative is equal to the Lipshitz constant. We shall prove only that $C_{f, \varepsilon}^{(s)+1}$ is a Banach space. The proof for $C_{f, \varepsilon}^{s}$ is standard.

Suppose that $\rho_{n}$ is a Cauchy sequence in the norm $\|\cdot\|_{(s)+1}$. Thus it is Cauchy in the norm $\|\cdot\|_{s}$ and therefore it converges to some $\rho$ in this norm. Let us take small $\gamma>0$ and $n_{0}$ such that for $n, m>n_{0},\left|\rho_{n}-\rho_{m}\right|_{(s)+1}<\gamma$. Hence

$$
\left|\rho_{n}^{(s+1)}-\rho_{m}^{(s+1)}\right|<\gamma \cdot \varphi_{s+1} \quad \text { a.e. }
$$

Define for $k \in \mathbb{N}$ and $\delta>1$

$$
V_{k}=\left\{x \in I \backslash B_{0}: \delta^{k-1} \leq \varphi_{s+1}(x)<\delta^{k}\right\}
$$

We have $\bigcup_{k=1}^{+\infty} V_{k}=I \backslash B_{0}$. Let $K$ be a component of $V_{k}$. Then $\left|\rho_{n}^{(s+1)}-\rho_{m}^{(s+1)}\right|<\gamma \cdot \delta^{k}$ a.e. on $K$. Hence

$$
\operatorname{Lip}\left(\left.\left(\rho_{n}-\rho_{m}\right)^{(s)}\right|_{K}\right) \leq \gamma \delta^{k}
$$

Since $\left.\rho_{n}^{(s)}\right|_{K}$ converges uniformly to $\left.\rho^{(s)}\right|_{K}$, Lip $\left(\left.\left(\rho^{(s)}-\rho_{m}^{(s)}\right)\right|_{K}\right) \leq \gamma \delta^{k}$. Hence $\left.\rho^{(s)}\right|_{K}$ is Lipshitz, because $\left.\rho_{m}^{(s)}\right|_{K}$ is Lipshitz. Consequently, $\rho^{(s)}$ is differentiable a.e. on $K$ and

$$
\left|\rho^{(s+1)}-\rho_{m}^{(s+1)}\right| \leq \gamma \delta^{k} \leq \gamma \delta \varphi_{s+1} \quad \text { a.e. on } K .
$$

Since this holds for all components $K$ of all $V_{k}$, it gives

$$
\frac{\left|\rho^{(s+1)}-\rho_{m}^{(s+1)}\right|}{\varphi_{s+1}} \leq \gamma \delta \quad \text { a.e. }
$$

Since $\delta$ was arbitrary, we have for $m>n_{0},\left|\rho-\rho_{m}\right|_{(s)+1} \leq \gamma$ and this proves (b). 
(c) Take $R=\left\{\rho \in C_{f, \varepsilon}^{(0)+1}:\|\rho\|_{(0)+1} \leq 1\right\}$. Let $\left\{\rho_{n}\right\}_{n \in N}$ be a sequence, $\rho_{n} \in R$. Notice that $v_{k}=I \backslash U_{1 / k}$ is compact, $k \in \mathbb{N}$, (see $\left.\S 4\right)$ and $\left.\varphi_{\varepsilon}\right|_{v_{k}}$ and $\left.\varphi_{1}\right|_{v_{k}}$ are bounded. Hence by the Ascoli-Arzela theorem the families $\left\{\left.\rho_{n}\right|_{v_{k}}\right\}_{n \in \mathbb{N}}, k \in \mathbb{N}$, are compact.

We can now choose a diagonal subsequence $\rho_{n}$ which converges pointwise and uniformly on compact subsets of $J \in \mathscr{J}$ to some $\rho$. Let $x \in V_{k}$ (defined for $\varphi_{\varepsilon}$ instead of $\left.\varphi_{s+1}\right)$, then we have

$$
|\rho(x)| \underset{1 \rightarrow+\infty}{\leftarrow}\left|\rho_{n_{l}}(x)\right| \leq\left\|\rho_{n_{l}}\right\|_{\varepsilon} \cdot \varphi_{\varepsilon}(x) \leq \delta^{k}=\delta \cdot \delta^{k-1} \leq \delta \varphi_{\varepsilon}(x) .
$$

Hence $\|\rho\|_{\varepsilon} \leq \delta$ and consequently since $\delta>1$ was arbitrary, $\|\rho\|_{\varepsilon} \leq 1$.

Let $V$ be a component of $V_{k}$ (defined for $\left.\varphi_{1}\right)$. Since $\left\|\rho_{n_{l}}\right\|_{(0)+1} \leq 1, \operatorname{Lip}\left(\left.\rho_{n_{l}}\right|_{V}\right) \leq \delta^{k}$. Hence also Lip $\left(\left.\rho\right|_{v}\right) \leq \delta^{k}$ and $\left|\left(\left.\rho^{\prime}\right|_{V}\right)(x)\right| \leq \delta \varphi_{1}(x)$ a.e. on $V$. Since $\delta>1$ was arbitrary we obtain essup $\left|\rho^{\prime}\right| / \varphi_{1} \leq 1$. Thus we have proved that $\rho \in R$. It remains to prove that $\rho_{n_{1}}$ converges to $\rho$ in $L_{1}(\lambda)$.

Take an $\hat{\varepsilon}>0$ and take $k_{0} \in \mathbb{N}$ such that $\int_{U_{1 / k_{0}}} \varphi_{\varepsilon} d \lambda<\hat{\varepsilon} / 3$. Then take $N \in \mathbb{N}$ such that $\left|\rho_{n_{l}}\right|_{v_{k_{0}}}-\left.\rho\right|_{v_{k_{0}}} \mid<\hat{\varepsilon} / 3$ for $l>N$. For $l>N$ this yields

$$
\begin{aligned}
\left\|\rho_{n_{t}}-\rho\right\|_{L_{1}} & \leq \int_{U_{1 / k_{0}}}\left|\rho_{n_{l}}\right| d \lambda+\int_{U_{1 / k_{0}}}|\rho| d \lambda+\int_{v_{k_{0}}}\left|\rho_{n_{t}}-\rho\right| d \lambda \\
& <\frac{\hat{\varepsilon}}{3}+\frac{\hat{\varepsilon}}{3}+\frac{\hat{\varepsilon}}{3}=\hat{\varepsilon} .
\end{aligned}
$$

This proves the compactness of $R$ in $L_{1}(\lambda)$.

Proposition 4.4. Let $f \in \mathcal{M}^{r}$. Then for each $\gamma>0$ there exist $G>0$ and $0<g<1$ such that if $\rho \in C_{f, \varepsilon}^{s}, s<r$, then for $x \notin U_{\gamma}$

$$
\left|\left(f_{*}^{n}(\rho)\right)^{(s)}(x)\right| \leq G\left(\mathrm{~g}^{n} \cdot|\rho|_{1, s}+\|\rho\|_{\varepsilon}\right) .
$$

Proof. From lemma 1.2 and lemma 4.2 we have

$$
\begin{aligned}
\left|\left(f_{*}^{n}(\rho)\right)^{(s)}(x)\right| & \leq C \sum_{i=0}^{s} f_{(i)}^{n}\left(\left|\rho^{(i)}\right|\right)(x) \\
& \leq C \sum_{i=1}^{s}|\rho|_{i} \cdot f_{(i)}^{n}\left(\varphi_{i}\right)(x)+C \cdot\|\rho\|_{\varepsilon} \cdot f_{*}^{n}\left(\varphi_{\varepsilon}\right)(x) \\
& \leq C|\rho|_{1, s} \sum_{i=1}^{s} f_{(i)}^{n}\left(\varphi_{i}\right)(x)+C\|\rho\|_{\varepsilon} f_{*}^{n}\left(\varphi_{\varepsilon}\right)(x)
\end{aligned}
$$

where $C=C(\gamma)$. Then applying lemma 3.4 and proposition 3.3 we obtain

$$
\begin{aligned}
\left|f_{*}^{n}(\rho)^{(s)}(x)\right| & \leq C\left(|\rho|_{1, s} \sum_{i=1}^{s} D(i) c^{n}+\|\rho\|_{\varepsilon}\left(\int \varphi_{\varepsilon} d \lambda / \gamma\right)\right) \\
& \leq G\left(g^{n} \cdot|\rho|_{1, s}+\|\rho\|_{\varepsilon}\right),
\end{aligned}
$$

where $g=\max _{i=1, \ldots, r-1} c(i)$ and $G=C \max \left\{\int \varphi_{\varepsilon} d \lambda / \gamma,(r-1) \cdot \max _{i=1,2, \ldots, r-1} D(i)\right\}$.

Lemma 4.2 and proposition 3.3 allow us to prove that the densities of invariant measures for $f$ are of class $C^{(r-2)+1}$ on compact subsets of $J \in \mathscr{I}$. Nevertheless we are going to investigate more precisely the Perron-Frobenius operator in the spaces defined in this section. 


\section{Global estimates}

In this section we assume $f$ belongs to $\mu$. Let us take $\delta>0$ such that for each $a \in \hat{A}, F(a, \delta) \subset U_{a}$ and

$$
\operatorname{dist}\left(W_{\delta}, \hat{B}\right)>\delta
$$

(see $\S 2(\mathrm{v}),(2.0)$ and the definition of distance). We shall often write $W$ instead of $W_{\delta}$. For each $n \in \mathbb{N}$ define a partition $\left\{U_{i}\right\}_{i=0}^{n}$ of $I$ :

$$
\begin{aligned}
& U_{i}=\left\{x \in \hat{I}: f^{i-1}(x)^{\vee} \in W \text { and }\left(f^{j}(x)\right)^{\vee} \notin W \text { for } j=i, \ldots, n-1\right\}^{\vee},(i=1, \ldots, n) ; \\
& U_{0}=E_{n}, \quad \text { where for } k \in \mathbb{N}, E_{k}=\left\{x \in \hat{I}:\left(f^{j}(x)\right)^{\vee} \notin W \text { for } j=0,1, \ldots, k-1\right\}^{\vee} .
\end{aligned}
$$

Lemma 5.1. For $i \in \mathbb{N} \cup\{0\}$ and $0<\eta<1$ there exist constants $G>0,0<h<1$ such that for $b \in \hat{B}, k \in \mathbb{N}$

$$
\left(\left.f^{k}\right|_{E_{k}}\right)_{(i)}\left(\psi_{b}^{\eta+i}\right) \leq G\left(\left|\left(f^{k}\right)^{\prime}(b)\right|^{\eta-1} \cdot \psi_{f^{k}(b)}^{\eta+i}+h^{k}\right)
$$

and for $k \in \mathbb{N}$

$$
\left(\left.f^{k}\right|_{E_{k}}\right)_{(i)}(1) \leq G \cdot h^{k}
$$

Proof. We shall write $\psi^{\zeta}$ instead of $\psi_{b}^{\zeta}$. In view of theorem 2.1 there exists $m \in \mathbb{N}$ such that $\left|\left(f^{m}\right)^{\prime}\right|_{E_{m}} \mid>\hat{\alpha}>1$.

(5.3) We extend $\left.f^{m}\right|_{E_{m}}$ to $g: E \rightarrow I$ such that $E=\bar{E}, E \supset E_{m}$ and $E$ is equal to a finite sum of intervals. Moreover, $\left|g^{\prime}\right|>\hat{\alpha}>1, \ln \left|g^{\prime}\right|$ is Lipshitz and for any component $K$ of $E, g(K)=I$.

Following (2.5) in [6], there is $\rho>0$ such that

$$
\left|\frac{\left(g^{k}\right)^{\prime}(x)}{\left(g^{k}\right)^{\prime}(y)}\right| \leq \rho
$$

if $x, y$ belong to the same component of $H_{k}$, where $H_{k}$ is the domain of $g^{k}$. Proposition 2.1 of [6] gives

$$
\lambda\left(H_{k}\right) \leq \beta^{k} \cdot \lambda(I) \quad 0<\beta<1 .
$$

Let us estimate the integral of $\psi^{\zeta}, 0<\zeta<1$ (we assume that $\underline{b}=-$ )

$$
\begin{aligned}
\int_{H_{k}} \psi^{\zeta} d \lambda & \leq \int_{\check{b}-\lambda\left(H_{k}\right)}^{\check{b}} \psi^{\zeta} d \lambda=\lambda\left(H_{k}\right)^{1-\zeta} /(1-\zeta) \\
& \leq \frac{\lambda(I)^{1-\zeta}}{1-\zeta}\left(\beta^{1-\zeta}\right)^{k}
\end{aligned}
$$

(when $\underline{b}=+$, the same inequality holds).

Let $K_{k}$ be the component of $H_{k}$ which contains $b$ and $\mathscr{K}_{k}\left(\mathscr{K}_{k}^{\prime}\right)$ be the set of all components of $H_{k} \backslash K_{k}\left(H_{k}\right)$. Put

$$
S=\max _{K \in \mathscr{K}_{1}^{\prime}} \sup _{x, y \in K} \sup _{z \in H_{1} \backslash K} \frac{\operatorname{dist}(x, z)}{\operatorname{dist}(y, z)} .
$$

We shall prove by induction that for each $k \in \mathbb{N}$

$$
\sup _{x, y \in K} \frac{\operatorname{dist}(x, \check{b})}{\operatorname{dist}(y, \check{b})} \leq \rho^{2} S \quad \text { for } K \in \mathscr{K}_{k} \text {. }
$$

From the definition of $S,(5.7)$ holds for $k=1$. 
Let (5.7) hold for some $k \in \mathbb{N}$. We consider $K^{\prime} \in \mathscr{K}_{k+1}$ such that $K^{\prime} \subset K \in \mathscr{K}_{k}$. Then the ratio in (5.7) for $K^{\prime}$ is less than or equal to the ratio for $K$. It remains to consider $K^{\prime} \in \mathscr{K}_{k+1}, K^{\prime} \subset K_{k}$. Notice that $K^{\prime \prime}=g^{k}\left(K^{\prime}\right) \in \mathscr{K}_{1}^{\prime}($ see $(5.3))$ and $K^{\prime \prime \prime}=g^{k}\left(K_{k+1}\right) \in \mathscr{K}_{1}$. Let $x_{0} \in K_{k}$, then by 5.4 for $x, y \in K^{\prime}$

$$
\frac{\operatorname{dist}(x, \check{b})}{\operatorname{dist}(y, \check{b})} \leq \frac{\rho}{\left|\left(g^{k}\right)^{\prime}\left(x_{0}\right)\right|} \operatorname{dist}\left(g^{k}(x), g^{k}(\check{b})\right):\left[\frac{1}{\rho\left|\left(g^{k}\right)^{\prime}\left(x_{0}\right)\right|} \operatorname{dist}\left(g^{k}(y), g^{k}(\check{b})\right)\right] \leq \rho^{2} S \text {. }
$$

Thus (5.7) is proved. From (5.7) it follows immediately that

$$
\psi(x) \leq \rho^{2} S \psi(y) \quad \text { for } x, y \in K, K \in \mathscr{K}_{k}, k \in \mathbb{N} .
$$

Let us prove the following:

(5.9) For each $0<\zeta<1$ and $i \in \mathbb{N}$ there exist $Q>0,0<q<1$ such that

$$
\left(\left.g^{k}\right|_{H_{k}-K_{k}}\right)_{(i)}\left(\psi^{\zeta+i}\right)(x) \leq Q q^{k} .
$$

Let $\phi(\zeta, i, K, x)=\left(g^{k} \mid K\right)_{(i)}\left(\psi^{\zeta+i}\right)(x), K \in \mathscr{K}_{k}$. We notice that

$$
\Theta(k, \zeta, i, x)=\sum_{K \in \mathscr{K}_{k}} \phi(\zeta, i, K, x)
$$

is equal to the left-hand side of (5.9).

We shall prove (5.9) for $i=0$ first. By (5.8) and (5.4)

and hence

$$
\phi(\zeta, 0, K, x) \leq \rho\left(\rho^{2} S\right)^{\zeta}: \phi(\zeta, 0, K, y) \quad K \in \mathscr{K}_{k}, x, y \in I
$$

$$
\Theta(k, \zeta, 0, x) \leq \rho\left(\rho^{2} S\right)^{\eta} \cdot \Theta(k, \zeta, 0, y)
$$

Since the Perron-Frobenius operator preserves integrals,

$$
\int_{I} \Theta \lambda(d x)=\int_{H_{k}-K_{k}} \psi^{\zeta} d \lambda
$$

Hence, by (5.6) for each $k \in \mathbb{N}$, one can find $x_{k}$ such that

$$
\Theta\left(k, \zeta, 0, x_{k}\right) \leq \frac{\left(\beta^{1-\zeta}\right)^{k}}{1-\zeta} \cdot \lambda(I)^{1-\zeta}
$$

Then, using (5.10) we obtain for every $x \in I$

$$
\Theta(k, \zeta, 0, x) \leq \rho\left(\rho^{2} S\right)^{\zeta} \Theta\left(k, \zeta, 0, x_{k}\right) \leq Q q^{k},
$$

where $Q=\rho\left(\rho^{2} S\right)^{\zeta} \cdot \lambda(I)^{1-\zeta} / 1-\zeta$ and $q=\beta^{1-\zeta}<1$. Hence (5.9) follows for $i=0$. Let us consider the case $i \neq 0$. Then using (5.11) for $\zeta=\xi$ we get

$$
\begin{aligned}
\phi(\zeta, i, K, x) & =\psi^{i+\zeta}(y) \cdot\left|\left(g^{k}\right)^{\prime}(y)\right|^{-i-1} \\
& =\left(\frac{\psi^{\xi}(y)}{\left|\left(g^{k}\right)^{\prime}(y)\right|}\right)^{i+1}=\phi(\xi, 0, K, x)^{i+1} \\
& \leq \phi(\xi, 0, K, x) \cdot \Theta(k, \xi, 0, x)^{i} \\
& \leq \phi(\xi, 0, K, x) \cdot H \cdot h^{k},
\end{aligned}
$$

where $\xi=(i+\zeta) /(i+1)<1, \quad K \in \mathscr{K}_{k}, \quad y \in K, \quad f^{k}(y)=x, \quad H=\left[\rho\left(\rho^{2} S\right)^{\xi} /((1-\xi)\right.$. $\left.\left.\lambda(I)^{1-\xi}\right)\right]^{i}$ and $h=\left(\beta^{1-\xi}\right)^{i}<1$. Immediately from (5.11) and (5.12) by taking the sum term by term it follows that

$$
\Theta(k, \zeta, i, x) \leq \Theta(k, \xi, i, x) \cdot H \cdot h^{k} \leq \underline{Q q^{k}},
$$

where $\underline{Q}=H^{2}$ and $\underline{q}=h \cdot \beta^{1-\xi}<1$. Hence (5.9) follows for $i \neq 0$. 
It remains to prove (5.14) and to deduce the lemma. Let $L_{l}$ be the component of $E_{l}$ containing $\check{b} .\left.f^{l}\right|_{L_{l}}$ is one to one.

Let $x=f^{l}(y), y \in L_{l} \cap \operatorname{supp} \psi_{b}, i \in \mathbb{N} \cup\{0\}, 0<\eta<1$. Then there exists $\tau>0$ such that

$$
\left(\left.f^{l}\right|_{L_{l}}\right)_{(i)}\left(\psi_{b}^{\eta+i}\right)(x) \leq \tau\left|\left(f^{l}\right)^{\prime}(b)\right|^{\eta-1} \cdot \psi_{f^{l}(b)}^{\eta+i}(x) .
$$

The left-hand side of (5.13) equals

$$
\left(|y-\check{b}|^{\eta+i}\left|\left(f^{\prime}\right)^{\prime}(y)\right|^{i+1}\right)^{-1}
$$

then by lemma $2.2 \mathrm{a}$ it is less than or equal to

$$
\left(\left(\frac{\left|x-\left(f^{l}(b)\right)^{\vee}\right|}{\rho\left|\left(f^{\prime}\right)^{\prime}(b)\right|}\right)^{\eta+i} \cdot\left(\frac{1}{\rho}\left|\left(f^{l}\right)^{\prime}(b)\right|\right)^{i+1}\right)^{-1}
$$

and this is equal to the right-hand side of (5.13) for $\tau=\rho^{2 i+\eta+1}$. Similarly we can prove that

$$
\left(\left.g^{k}\right|_{K_{k}}\right)_{(i)}\left(\psi_{b}^{\eta+i}\right) \leq x\left|\left(g^{k}\right)^{\prime}(b)\right|^{\eta-1} \cdot \psi_{g^{k}(b)}^{\eta+i}
$$

Finally (5.9), (5.13) and (5.14) allow us to prove the lemma.

Take $n=k m+r, 0 \leq r<m, k \in \mathbb{N}$. Notice that $E_{n} \subset E_{k m} \subset H_{k}$. Then

$$
\begin{aligned}
\left(\left.f^{n}\right|_{E_{n}}\right)_{(i)} & =\left(\left.f^{r}\right|_{E_{r}}\right)_{(i)} \circ\left(\left.f^{k m}\right|_{E_{n}}\right)_{(i)} \\
& \leq\left(\left.f^{r}\right|_{E_{r}}\right)_{(i)} \circ\left(\left.f^{k m}\right|_{E_{k m}}\right)_{(i)} \\
& \leq\left(\left.f^{r}\right|_{E_{r}}\right)_{(i)} \circ\left(\left.g^{k}\right|_{H_{k}}\right)_{(i)}
\end{aligned}
$$

(5.9) and (5.14) give the estimates

$$
\begin{aligned}
\left(\left.g^{k}\right|_{H_{k}}\right)_{(i)}\left(\psi_{b}^{\eta+i}\right) & \leq\left(\left.g^{k}\right|_{H_{k}-K_{k}}\right)_{(i)}\left(\psi_{b}^{\eta+i}\right)+\left(\left.g^{k}\right|_{K_{k}}\right)_{(i)}\left(\psi_{b}^{\eta+i}\right) \\
& \leq Q q^{k}+\varkappa\left|\left(g^{k}\right)^{\prime}(b)\right|^{\eta-1} \cdot \psi_{g^{k}(b)}^{\eta+i} .
\end{aligned}
$$

Then, applying this to (5.15) we have

$$
\begin{aligned}
& \left(\left.f^{n}\right|_{E_{n}}\right)_{(i)}\left(\psi_{b}^{\eta+i}\right) \\
& \quad \leq Q q^{k}\left(\left.f^{r}\right|_{E_{r}}\right)_{(i)}(1)+\varkappa\left|\left(g^{k}\right)^{\prime}(b)\right|^{\eta-1}\left[\left(\left.f^{r}\right|_{E_{r}-L_{r}}\right)_{(i)}\left(\psi_{g^{k}(b)}^{\eta+i}\right)+\left(\left.f^{r}\right|_{L_{r}}\right)_{(i)}\left(\psi_{g^{k}(b)}^{\eta+i}\right)\right] .
\end{aligned}
$$

Now using (5.13) for $l=r$ we have (notice that $f^{n}(x)=f^{r} \circ g^{k}(x)$, for $x \in E_{n}$ ):

$$
\begin{aligned}
\left(\left.f^{n}\right|_{E_{n}}\right)_{(i)}\left(\psi_{b}^{\eta+i}\right) & \leq Q q^{k} C_{1}+\chi\left(\hat{\alpha}^{\eta-1}\right)^{k} C_{2}+\varkappa \tau\left|\left(f^{n}\right)^{\prime}(b)\right|^{\eta-1} \cdot \psi_{f^{n}(b)}^{\eta+i} \\
& \leq G\left(h^{n}+\left|\left(f^{n}\right)^{\prime}(b)\right|^{\eta-1} \psi_{f^{n}(b)}^{\eta+i}\right)
\end{aligned}
$$

where

$$
\begin{aligned}
& C_{1}=\sup _{x \in I, r<m}\left(\left.f^{r}\right|_{E_{r}}\right)_{(i)}(1)(x)<+\infty ; \\
& C_{2}=\sup _{x \in I, r<m, a \in \hat{B}}\left(\left.f^{r}\right|_{E_{r}-L_{r}}\right)_{(i)}\left(\psi_{a}^{\eta+i}\right)(x)<+\infty ; \\
& G=\max \left\{Q \cdot C_{1} / q, x C_{2} / \hat{\alpha}^{\eta-1}, x \tau\right\} ;
\end{aligned}
$$

and

$$
h=\max \left\{q^{1 / m}, \hat{\alpha}^{(\eta-1) / m}\right\}<1 .
$$

This finishes the proof of the first inequality. A proof of the second inequality is actually contained in the above argument. 
LemMA 5.2. Let $f$ belong to $\mathcal{M}^{r}$. There exist constants $H>0,0<g<1$ ( $g$ being the same as in proposition 4.4) such that for $i=1,2, \ldots, n$ and $\rho \in C_{f, \varepsilon}^{s}, 0 \leq s<r$,

$$
\left|\left(\left.f^{i}\right|_{U_{i}}\right)_{*}(\rho)^{(s)}\right| \leq H\left(g^{i} \cdot|\rho|_{1, s}+\|\rho\|_{\varepsilon}\right) \cdot \sum_{a \in \hat{A}_{2}} \psi_{f(a)}^{\xi(a)+s}
$$

$(\xi(a)$ is from $(3.10))$.

Proof. Let $F_{a}=F(a, \delta)\left(=W_{\delta} \cap U_{a}\right)$ for $a \in \hat{A}_{2}\left(\delta\right.$ is from (5.1)). Set $U_{i, a}=$ $\left\{x \in U_{i}: f^{i-1}(x) \in F_{a}\right\}$. Then

$$
\left(\left.f^{i}\right|_{U_{i}}\right)_{*}=\sum_{a \in \hat{A}_{2}}\left(\left.f^{i}\right|_{U_{i, a}}\right)_{*}
$$

From (v) and (5.1) it follows that $U_{\delta} \cap W_{\delta}=\varnothing$. Therefore, applying proposition 4.4 one can write for $x \in \hat{I}$

$$
\left|\left(\left.f^{i-1}\right|_{U_{i, a}}\right)_{*}(\rho)^{(j)}(x)\right| \leq G\left(g^{i-1}|\rho|_{1, s}+\|\rho\|_{\varepsilon}\right) \quad j \leq s .
$$

(In fact here we use a slightly stronger result than proposition 4.4. This is that the sum of the absolute values of the derivatives of all terms of $f_{*}^{n}(\rho)$ is less than or equal to the expression stated in proposition 4.4. It is proved within the proof of proposition 4.4. Taking above the restriction $\left.f^{i-1}\right|_{U_{i, a}}$ instead of $f^{i-1}$ we omit some terms for given $x$ and the estimate remains true.)

Let $\rho_{1}=\left(\left.f^{i-1}\right|_{U_{i, a}}\right)_{*}(\rho)$ and $q=\left.f\right|_{F_{a}}$, (we observe that $q$ is one-to-one). Then

$$
\left(\left.f^{i}\right|_{U_{i, a}}\right)_{*}(\rho)=q_{*}\left(\rho_{1}\right)
$$

From lemma 1.1 we obtain:

$$
\left|q_{*}\left(\rho_{1}\right)^{(j)}(x)\right| \leq \sum_{k=0}^{j} \sum_{Z \in \mathscr{Z}\left(k, j, q^{-1}\right)}|S(k, Z)|,
$$

and

$$
S(k, Z)(x)=D^{k}(x) \prod_{(l, 1, p) \in Z} D_{1}^{l}(x)^{p}
$$

Let us estimate $D_{1}^{l}$ and $D^{k}$ (see lemma 1.1 and conditions (vi), (vi')). Let $q(y)=x$, then

$$
\left|D_{1}^{l}(x)\right| \leq \frac{\kappa \cdot|y-\check{a}|^{v}}{\left(\alpha|y-\check{a}|^{u}\right)^{l+1}}=\frac{\kappa}{\alpha^{l+1}}|y-\check{a}|^{v-u \cdot(l+1)} .
$$

Since $v \geq u-l\left(\left(\mathrm{vi}^{\prime}\right)\right)$, for some constant $E_{1} \geq \kappa / \alpha^{l+1}$ using (2.1) we have

$$
\begin{aligned}
\left|D_{1}^{l}(x)\right| & \leq E_{1}|y-\check{a}|^{u-l-u \cdot(l+1)}=E_{1}|y-\check{a}|^{-l \cdot(u+1)} \\
& \leq E_{2}(l) \cdot\left|x-(f(a))^{\vee}\right|^{-l}
\end{aligned}
$$

where $E_{2}(l)=E_{1}(\omega /(u+1))$.

We now estimate $D^{k}$ using (5.17), (vi) and (2.1)

$$
D^{k}(x)=\frac{\sup \left|\rho_{1}^{(k)}\right|_{F_{a}} \mid}{\left(\alpha|y-\check{a}|^{u}\right)^{k+1}} \leq \frac{G\left(g^{i-1}|\rho|_{1, s}+\|\rho\|_{\varepsilon}\right)}{E_{3}\left|x-(f(a))^{v}\right|^{\xi(a) \cdot(k+1)}}
$$

where $E_{3}=\alpha^{k+1}((u+1) / \omega)^{\xi(a) \cdot(k+1)}$.

$(5.18)-(5.20)$ give us

$$
|S(k, Z)(x)| \leq E_{4}\left(g^{i} \cdot|\rho|_{1, s}+\|\rho\|_{\varepsilon}\right) \cdot \psi_{f(a)}^{p(a)},
$$


where $E_{4}=\max _{a \in \hat{A}_{2}} \max _{Z \in \mathscr{X}\left(k, j, q^{-1)}\right.}\left(\left(G / E_{3} g\right) \prod_{(l, 1, p) \in Z} E_{2}(l)^{p}\right)$ and

$$
\begin{aligned}
p(a) & =\xi(a)+k \xi(a)+\sum_{(l, 1, p) \in Z} l p \\
& \leq \xi(a)+k+\sum_{(l, 1, p) \in Z} l \cdot p=\xi(a)+j
\end{aligned}
$$

(see lemma 1.1 ), since $k+\sum_{(l, 1, p) \in Z} l \cdot p=j, 0 \leq l \leq j$. Hence also card $\mathscr{Z}\left(k, j, q^{-1}\right) \leq 2^{j}$. Therefore from (5.18), (5.21) it follows that

$$
\left|\left(\left.f^{i}\right|_{U_{i, a}}\right)_{*}(\rho)^{(j)}\right| \leq H\left(g^{i}|\rho|_{1, s}+\|\rho\|_{\varepsilon}\right) \cdot \psi_{f(a)}^{\xi(a)+j},
$$

where $H=(j+1) \cdot 2^{j} \cdot E_{4}$. Now the lemma follows from (5.16).

LEMMA 5.3. There exists a constant $H>0$ such that

$$
\left(\left.f^{i}\right|_{U_{i}}\right)_{*}\left(\varphi_{\varepsilon}\right) \leq H \sum_{a \in \hat{A}_{2}} \psi_{f(a)}^{\xi(a)} \quad \text { for } i=1, \ldots, n
$$

Proof. One can easily prove this from proposition 3.3 and (vi). We omit the precise proof since it is actually a part of the proof of lemma 5.2 .

Now we have a method to prove the fundamental proposition of this paper.

Proposition 5.4. (a) There exist constants $\Theta>0$ and $\vartheta<1$ such that for $n \in \mathbb{N}$

$$
f_{*}^{n}\left(\varphi_{\varepsilon}\right) \leq \Theta\left(\varphi_{0}+\vartheta^{n} \varphi_{\varepsilon}\right) \text { and }\left\|f_{*}^{n}\right\|_{\varepsilon} \leq 2 \Theta \text {. }
$$

(b) If $f \in M^{r}$ and $\varepsilon>0$ then there exist constants $T>0$ and $\tau<1$ such that for $n \in \mathbb{N}, \rho \in C_{f, \varepsilon}^{s}$ and $1 \leq s<r$,

(if $\varepsilon=0$ then $\tau=1$ ).

$$
\left|f_{*}^{n}(\rho)\right|_{1, s} \leq T\left(\tau^{n} \cdot|\rho|_{1, s}+\|\rho\|_{\varepsilon}\right) ;
$$

Proof. We notice first that $\left.f^{n}\right|_{U_{i}}=\left.\left.f^{n-i}\right|_{E_{n-i}} \circ f^{i}\right|_{U_{i}}$ and hence

$$
\left(\left.f^{n}\right|_{U_{i}}\right)_{*}=\left(\left.f^{n-i}\right|_{E_{n-i}}\right)_{*} \circ\left(\left.f^{i}\right|_{U_{i}}\right)_{*} .
$$

Then we notice that since $\bigcup_{i=0}^{n} U_{i}=I$,

$$
\sum_{i=0}^{n}\left(\left.f^{n}\right|_{U_{i}}\right)_{*}=f_{*}^{n}
$$

(a) By lemma 5.3 and (5.22), since $f^{i}\left(U_{i}\right) \subset W$, we have, $0<i \leq n$,

$$
\left(\left.f^{n}\right|_{U_{i}}\right)_{*}\left(\varphi_{\varepsilon}\right) \leq H\left(\left.f^{n-i}\right|_{E_{n-i}}\right)_{*}\left(\sum_{a \in \hat{A}_{2}} \psi_{f(a)}^{\xi(a)}\right) .
$$

Hence by lemma 5.1 it is less than or equal to

$$
G H\left(\sum_{a \in \hat{A}_{2}}\left|\left(f^{n-i}\right)^{\prime}(f(a))\right|^{\xi(a)-1} \cdot \psi_{f^{n-i+1}(a)}^{\xi(a)}+h^{n-i}\right) .
$$

Then by lemma 5.1 and (3.10),

$$
\begin{aligned}
\left(\left.f^{n}\right|_{U_{0}}\right)_{*}\left(\varphi_{\varepsilon}\right) & \leq G \sum_{k=0}^{+\infty} \sum_{a \in \hat{A}_{2}}\left|\left(f^{k}\right)^{\prime}\left(a_{1}\right)\right|^{-\eta(a)}\left[\left|\left(f^{n}\right)^{\prime}\left(a_{k+1}\right)\right|^{\xi(a)+\varepsilon-1} \cdot \psi_{a_{n+k+1}}^{\xi(a)+\varepsilon}+h^{k}\right]+G h^{n} \\
& =G\left[\sum_{k=0}^{+\infty} \sum_{a \in \hat{A}_{2}}\left|\left(f^{n}\right)^{\prime}\left(a_{k+1}\right)\right|^{-\varepsilon} \cdot\left|\left(f^{n+k}\right)^{\prime}\left(a_{1}\right)\right|^{-\eta(a)} \cdot \psi_{a_{n+k+1}}^{\xi(a)+\varepsilon}+E_{1} h^{k}\right],
\end{aligned}
$$


where $E_{1}=\sum_{k=0}^{+\infty} \sum_{a \in \hat{A}_{2}}\left|\left(f^{k}\right)^{\prime}\left(a_{1}\right)\right|^{-\eta(a)}+1<+\infty$. Since $\left|\left(f^{k}\right)^{\prime}\left(a_{1}\right)\right|$ grows exponentially, one can find constants $E_{2}>0$ and $h_{1}<1$ such that

$$
\left|\left(f^{n}\right)^{\prime}\left(a_{k+1}\right)\right|^{-\varepsilon}<E_{2} h_{1}^{n} \quad \text { for } k, n \in \mathbb{N} .
$$

Now (5.25) gives us:

$$
\left(\left.f^{n}\right|_{U_{0}}\right)_{*}\left(\varphi_{\varepsilon}\right) \leq E_{3} h_{2}^{n} \varphi_{\varepsilon}
$$

where $E_{3}=G \cdot \max \left\{E_{1}, E_{2}\right\}$ and $h_{2}=\max \left\{h_{1}, h\right\}<1$.

Let us look at (5.24). We can write $-\eta(a)$ instead of $\xi(a)-1$ (maybe making $G H$ larger) which is less than $-\eta(a)$. This is possible since $\left|\left(f^{k}\right)^{\prime}(b)\right|>1$ for $b \in \hat{B}$ and for $k$ large enough.

Let us sum (5.24) for $i=1, \ldots, n$ and then add (5.26) to the resulting inequality. In view of (5.23), we have (a) for $\Theta=\max \left\{G H, G H /(1-h), E_{3}\right\}$ and $\vartheta=h_{2}<1$.

In order to estimate $\left\|f_{*}^{n}\right\|_{\varepsilon}$ we notice that $|\rho| \leq\|\rho\|_{\varepsilon} \cdot \varphi_{\varepsilon}$. Hence, by the first part,

$$
\left|f_{*}^{n}(\rho)\right| \leq f_{*}^{n}(|\rho|) \leq\|\rho\|_{\varepsilon} \cdot \Theta\left(\varphi_{0}+\vartheta^{n} \varphi_{\varepsilon}\right) \leq\|\rho\|_{\varepsilon} 2 \Theta \varphi_{\varepsilon}
$$

(notice that if $\lambda(I) \leq 1$ then $\varphi_{\varepsilon} \geq \varphi_{0}$. If not then one has to increase $\Theta$ ).

(b) From now on $x \in \hat{I} \backslash \hat{B}$ and $\check{x} \notin B_{0}$. Let $\rho \in C_{f, \varepsilon}^{s}$. In view of (5.22), (5.23),

$$
\begin{aligned}
f_{*}^{n}(\rho)^{(s)} & =\sum_{i=0}^{n}\left(\left.f^{n}\right|_{U_{i}}\right)_{*}(\rho)^{(s)} \\
& =\sum_{i=0}^{n}\left[\left(\left.f^{n-i}\right|_{E_{n-i}}\right)_{*} \circ\left(\left.f^{i}\right|_{U_{i}}\right)_{*}(\rho)\right]^{(s)} .
\end{aligned}
$$

Let $\rho_{i}=\left(\left.f^{i}\right|_{U_{i}}\right)_{*}(\rho)$. Then by remark 1.3

$$
\left|\left(\left.f^{n-i}\right|_{E_{n-i}}\right)_{*}\left(\rho_{i}\right)^{(s)}\right| \leq \sum_{j=0}^{s}\left(\left.f^{n-i}\right|_{E_{n-i}}\right)_{(j)}\left(\left|\rho_{i}^{(j)}\right|\right) \cdot T(j, s, n-i) .
$$

Theorem 2.1 easily implies the existence of constants $C>0$ and $\beta>1$ such that

$$
\left|\left(f^{k}\right)^{\prime}(x)\right| \geq C \cdot \beta^{k} \quad \text { for } x \in E_{l}, l \geq k \text {. }
$$

This enables us to follow the proof of lemma 4.1 and lemma 4.2 and to obtain:

(5.30) There exists a constant $D$ such that $T(j, s, n)(x)<D$ for $j \leq s<r, n \in \mathbb{N}$ and $x \in I$.

According to lemmas (5.1) and (5.2), and (5.29) we get (notice that $(f(\hat{A}))^{\vee} \subset E_{n}$ for each $n \in \mathbb{N}$ )

$$
\begin{aligned}
&\left(\left.f^{n-i}\right|_{E_{n-i}}\right)_{(j)}\left(\rho_{i}^{(j)}\right) \\
& \quad \leq H\left(g^{i} \cdot|\rho|_{1, j}+\|\rho\|_{\varepsilon}\right) \cdot\left(\left.f^{n-i}\right|_{E_{n-i}}\right)_{(j)}\left(\sum_{a \in \hat{A}_{2}} \psi_{f(a)}^{\xi(a)+j}\right) \\
& \quad \leq H\left(g^{i}|\rho|_{1, j}+\|\rho\|_{\varepsilon}\right) \sum_{a \in \hat{A}_{2}} G\left(\left|\left(f^{n-i}\right)^{\prime}(f(a))\right|^{\xi(a)-1} \cdot \psi_{f^{n-1}(f(a))}^{\xi(a)+j}+h^{n-i}\right) \\
& \quad \leq H_{1}\left(g^{i}|\rho|_{1, j}+\|\rho\|_{\varepsilon}\right) h_{1}^{n-i} \sum_{a \in \hat{A}_{2}}\left(\left|\left(f^{n-i}\right)^{\prime}(f(a))\right|^{-\eta(a)} \cdot \psi_{f^{n-i}(f(a))}^{\xi(a)+j}+1\right),
\end{aligned}
$$

where $H_{1}=H G \max \left\{C^{-2 \varepsilon}, 1\right\}$ and $h_{1}=\max \left\{\beta^{-2 \varepsilon}, h\right\}<1$. 
Let us return now to (5.27), (5.28) and there apply (5.30) and (5.23).

$$
\begin{aligned}
\left|f_{*}^{n}(\rho)^{(s)}\right| & \leq D \sum_{j=0}^{s} \sum_{i=0}^{n}\left(\left.f^{n-i}\right|_{E_{n-i}}\right)_{(j)}\left(\left|\rho_{i}^{(j)}\right|\right) \\
& =D\left(f_{*}^{n}(|\rho|)+\sum_{j=1}^{s} S_{j}\right)
\end{aligned}
$$

where $S_{j}=\sum_{i=0}^{n}\left(\left.f^{n-i}\right|_{E_{n-i}}\right)_{(j)}\left(\left|\rho_{i}^{(j)}\right|\right)$. According to (5.31) and (3.10) for $g_{1}=$ $\max \left\{g, h_{1}\right\}<1$ we have

$$
\begin{aligned}
S_{j} \leq & H_{1}\left(g_{1}^{n}|\rho|_{1, j}+\|\rho\|_{\varepsilon}\right) \cdot\left(\varphi_{j}-1\right) \\
& +H_{1} \cdot \operatorname{card} \hat{A}_{2} \cdot \sum_{i=0}^{n}\left(g_{1}^{n} \cdot|\rho|_{1, j}+h^{n-i}\|\rho\|_{\varepsilon}\right) .
\end{aligned}
$$

Now since $|\rho|_{1, j} \leq|\rho|_{1, s}$ and for $g_{2}: g_{1}<g_{2}<1$ there exists $G_{2}$ such that $(n+1) g_{1}^{n} \leq$ $G_{2} g_{2}^{n}$ for $n \in \mathbb{N}$, we have

$$
\begin{aligned}
S_{j}(x) \leq & H_{1}\left(g_{1}^{n} \cdot|\rho|_{1, s}+\|\rho\|_{\varepsilon}\right) \cdot\left(\varphi_{j}(x)-1\right) \\
& +H_{1} \cdot \operatorname{card} \hat{A}_{2} \cdot\left[(n+1) g_{1}^{n}|\rho|_{1, s}+(1 / 1-h)\|\rho\|_{\varepsilon}\right] \\
\leq & H_{2}\left(g_{2}^{n}|\rho|_{1, s}+\|\rho\|_{\varepsilon}\right) \cdot \varphi_{j},
\end{aligned}
$$

where $H_{2}=H_{1} \cdot$ card $\hat{A}_{2} \cdot \max \left\{G_{2}, 1 / 1-h\right\}$. Having (5.33) and the second part of (a) we can apply (5.32) to obtain:

$$
\left|f_{*}^{n}(\rho)^{(s)}(x)\right| \leq D\left[2 \cdot \Theta \cdot\|\rho\|_{\varepsilon} \cdot \varphi_{\varepsilon}(x)+H_{2}\left(g_{2}^{n}|\rho|_{1, s}+\|\rho\|_{\varepsilon} \cdot \sum_{j=1}^{s} \varphi_{j}(x)\right] .\right.
$$

Dividing both sides by $\varphi_{s}$ and taking the supremum over $x \in J \in \mathscr{J}$ we have:

$$
\left|f_{*}^{n}(\rho)\right|_{s} \leq D\left[2 \Theta\|\rho\|_{\varepsilon} \cdot \alpha_{\varepsilon}+H_{2}\left(g_{2}^{n}|\rho|_{1, s}+\|\rho\|_{\varepsilon}\right) \sum_{j=1}^{s} \alpha_{j}\right],
$$

where $\alpha_{\varepsilon}=\sup \varphi_{\varepsilon} / \varphi_{s}<+\infty$ and $\alpha_{j}=\sup \varphi_{j} / \varphi_{s}<+\infty$. Hence proposition 5.4(b) follows for $\tau=g_{2}<1$ and $T=D\left(2 \Theta \alpha_{\varepsilon}+H_{2} \sum_{j=1}^{s} \alpha_{j}\right)$.

Remark 5.5(a) In proposition 5.4(b) the space $C_{f, \varepsilon}^{s}$ can be replaced by the space $C_{f, \varepsilon}^{(s-1)+1}$ and the semi-norm $|\cdot|_{1, s}$ by the semi-norm $|\cdot|_{1,(s-1)+1}$. Then the pattern of the proof remains the same. The only difference is that certain expressions hold almost everywhere (compare remark 4.3(a)).

(b) Proposition 5.4(a) implies the existence of absolutely continuous invariant measures for $f$. This gives the proof, different from that of M. Misiurewicz [6].

6. Main results in the class $\mathcal{M}^{r}$

In this section $f$ belongs to $\mathcal{M}^{r}(r \geq 2)$. Let us recall some results of $\S 6$ of [6].

There exists a finite family of pairs $\left\{\left(K_{i, j}, \nu_{i, j}\right)\right\}_{1 \leq j \leq k_{i}, 1 \leq i \leq p}$ satisfying (6.1)-(6.6). For $K_{i, j}$ we take $j$ modulo $k_{i}$.

(6.1) $f^{k_{i}}\left(K_{i, j}\right)=K_{i, j}$

(6.2) $K_{i, j} \subset I$ is a finite sum of closed intervals and the $K_{i, j}$ are pairwise disjoint;

(6.3) $\nu_{i, j} \in L_{1}(\lambda), \int \nu_{i, j} d \lambda=1$ and $\operatorname{supp}\left(\nu_{i, j}\right)=K_{i, j}$;

(6.4) $f\left(K_{i, j}\right)=K_{i, j+1}, f_{*}\left(\nu_{i, j}\right)=\nu_{i, j+1}$;

(6.5) $\left\{\left.f^{k_{i}}\right|_{K_{i, j}}, \nu_{i, j} d \lambda\right\}$ is exact; 
We define for each $K_{i j}$ the basin of $K_{i, j}$ :

$$
M_{i, j}=\bigcup_{n=0}^{+\infty} f^{-n k_{i}}\left(K_{i, j}\right)
$$

Then for $\rho \in L_{1}(\lambda)$ :

(6.6) $\lim _{n \rightarrow+\infty} f_{*}^{n k_{i}}\left(\chi_{M_{i, j}} \cdot \rho\right)=\nu_{i, j} \cdot \int_{M_{i, j}} \rho d \lambda$ in $L_{1}(\lambda)$.

For $\rho$ of class $C^{2}$ the convergence is also in the topology of uniform convergence on compact subsets of $J \in \mathscr{F}$. We remark that all results obtained for the partition $\mathscr{F}$ defined in $[6, \S 5]$ remain true for our partition $\mathscr{F}$ defined in $\$ 4$ above.

Let us define the projection

$$
\pi: L_{1}(\lambda) \rightarrow L_{1}(\lambda), \quad \pi(\rho)=\sum_{i, j} \nu_{i, j} \int_{M_{i, j}} \rho d \lambda .
$$

From (6.4) it follows that

$$
f^{-1}\left(M_{i, j}\right)=M_{i, j-1} \quad 1 \leq i \leq s, \quad 1 \leq j \leq k_{i} .
$$

Namely, $f^{-1}\left(M_{i, j}\right)=\bigcup_{n=0}^{+\infty} f^{-k_{i} n}\left(f^{-1}\left(K_{i, j}\right)\right)$. Now since

$$
f^{k_{i}}\left(f^{-1}\left(K_{i, j}\right)\right)=K_{i, j-1} \text { and } f^{-1}\left(K_{i, j}\right) \subset M_{i, j-1}
$$

(6.7) follows. This and (6.4) yield

$$
f_{*} \circ \pi=\pi \circ f_{*} \quad \text { and } f_{*}^{n} \circ \pi(\rho)=\sum_{i, j} \nu_{i, j+n} \int_{M_{i, j}} \rho d \lambda ;
$$

(notice that $\int_{M_{i, j}} f_{*}(\rho) d \lambda=\int_{f^{-1}\left(M_{i, j}\right)} \rho d \lambda=\int_{M_{i, j-1}} \rho d \lambda$ ). Let us define the remainder operator $\phi=f_{*}-f_{*} \circ \pi$. In view of $(6.8)$ since $\pi^{2}=\pi$,

$$
f_{*}^{n}=f_{*}^{n} \circ \pi+\phi^{n} \quad \text { for all } n \in \mathbb{N} \text {. }
$$

The following proposition is a consequence of (6.6).

Proposition 6.1. For each $\rho \in L_{1}(\lambda), \lim _{n \rightarrow+\infty}\left\|\phi^{n}(\rho)\right\|_{L_{1}}=0$.

Proof. Let $n_{0}=\prod_{i=1}^{p} k_{i}$. Then $f_{*}^{n_{0} l} \circ \pi=\pi$ and by (6.9):

$$
\begin{aligned}
\phi^{n_{0} l}(\rho) & =f_{*}^{n_{0} l}(\rho)-\pi(\rho) \\
& =\sum_{i, j}\left(f_{*}^{n_{0} l}\left(\chi_{M_{i, j}} \cdot \rho\right)-\nu_{i, j} \int_{M_{i, j}} \rho d \lambda\right)+f_{*}^{n_{0} l}\left(\chi_{M_{0}} \cdot \rho\right) \\
& =\phi^{n_{0} l}\left(\chi_{M} \cdot \rho\right)+f_{*}^{n_{0} l}\left(\chi_{M_{0}} \rho\right),
\end{aligned}
$$

where $M_{0}=I \backslash M$ and $M=\bigcup_{i, j} M_{i, j}$. We shall now prove that

$$
\lim _{l \rightarrow+\infty}\left\|\phi^{n_{0} l}(\rho)\right\|_{L_{1}}=0 \text {. }
$$

Let $\rho_{l}=\phi^{n_{0} l}(1)$ and let us consider the set

$$
H=\operatorname{cl} \operatorname{conv}\left\{\rho_{l}: l \in \mathbb{N}\right\} \text {. }
$$

In view of proposition 5.4 and theorem 6.2(c) of [6], $H$ is compact, the $L_{1}$ topology and topology of uniform convergence on compact subsets of $J \in \mathscr{J}$ (u.c.s. topology) coincide on $H$, and $H \subset \mathscr{D}_{0}$.

Let $\rho \equiv 1$ in (6.10) and let us suppose that there exists a subsequence $\rho_{l_{k}}$ of $\rho_{l}$ such that $\lim _{k \rightarrow+\infty} \rho_{l_{k}}=\varphi \not \equiv 0$. Since by (6.6) $\lim _{l \rightarrow+\infty} f_{*}^{n_{0} l}\left(\chi_{M}\right) \stackrel{L_{i}^{k}}{=} \pi(1)$, 
$\lim _{k \rightarrow+\infty} f_{*}^{n_{0} l_{k}}\left(\chi_{M_{0}}\right) \stackrel{L_{1}}{=} \varphi$. Since $\varphi \in H \subset \mathscr{D}_{0}$, there exists $J_{0} \in \mathscr{J}$ such that $\left.\varphi\right|_{J_{0}}>0$. Because $f\left(M_{0}\right) \subset M_{0}, J_{0}$ has to be contained in $M_{0}$. Take $M_{1}=\bigcup_{n=0}^{+\infty} f^{n}\left(J_{0}\right)$. We have $M_{1} \cap M=\varnothing, M_{1}$ is invariant under $f$ and is equal to the composition of elements of $\mathscr{J}$. Thus it has to contain some set $K_{i, j}$ (one can deduce a proof from $[6, \S 5])$. This leads to a contradiction, since no $K_{i, j}$ intersects $M_{0}\left(\supset M_{1}\right)$. Hence we have that $\varphi \equiv 0$ and (6.11) for $\rho \equiv 1$ follows. Now by (6.10), (6.11) we have

$$
\lambda\left(M_{0}\right)=\int \chi_{M_{0}} d \lambda=\int f_{*}^{n_{0} l}\left(\chi_{M_{0}}\right) d \lambda \underset{l \rightarrow+\infty}{\stackrel{L_{1}}{\rightarrow}} 0
$$

and $\lambda\left(M_{0}\right)=0$. This yields the identity $\phi^{n_{0} l}(\rho)=\phi^{n_{0} l}\left(\rho \cdot \chi_{M}\right)$ a.e. Simultaneously, (6.6) implies that

$$
\phi^{n_{0} l}\left(\chi_{M} \cdot \rho\right) \underset{l \rightarrow+\infty}{\stackrel{L_{1}}{\rightarrow}} 0 .
$$

Hence (6.11) follows. Now, since $\left\|\phi^{n}\right\|_{L_{1}} \leq 2$ for $n \in \mathbb{N}$ the proposition follows as well.

Remark 6.2. We have proved that $\lambda\left(M_{0}\right)=0$, which is necessary for theorem $6.3(\mathrm{~g}),(\mathrm{h})$ of [6]. M. Misiurewicz omitted this proof in [6].

We are now in a position to prove the main theorem.

TheOREM 6.3(a) $C_{f, \varepsilon}^{s}$ for $s=0,1, \ldots, r-1$ is invariant under $f_{*}$ and there exists $C>0$ such that:

$$
\begin{array}{ll}
\left\|f_{*}^{n}\right\|_{s} \leq C & \text { for } n \in \mathbb{N}, s=1, \ldots, r-1 ; \\
\left\|f_{*}^{n}\right\|_{\varepsilon} \leq C & \text { for } n \in \mathbb{N} .
\end{array}
$$

(b) $\nu_{i, j} \in C_{f, \varepsilon}^{r-1}$.

(c) $\pi, \phi: C_{f, \varepsilon}^{s} \supset$ are continuous $(s=0,1, \ldots, r-1)$.

(d) There exist canstants $\Gamma>0$ and $0<\gamma<1$ such that for $\rho \in C_{f, \varepsilon}^{(0)+1}$ and $n \in \mathbb{N}$,

$$
\left\|\phi^{n}(\rho)\right\|_{L_{1}} \leq \Gamma \gamma^{n} \cdot\|\rho\|_{(0)+1} \text {. }
$$

(e) For $\varepsilon>0$ assumed to be as in (3.10) there exist constants $\Lambda>0$ and $0<\varsigma<1$ such that

$$
\left\|\phi^{n}\right\|_{s} \leq \Lambda \cdot \varsigma^{n} \quad \text { for } n \in \mathbb{N}, s=1, \ldots, r-1 .
$$

(Briefly speaking, the spectral radius of $\phi$ in $C_{f, \varepsilon}^{s}$ is less than one.)

Proof. Again let $n_{0}=\prod_{i=1}^{p} k_{i \cdot}$. Then $f^{n_{0} l} \circ \pi=\pi$ and by proposition 6.1 for $\rho \in L_{1}(\lambda)$

$$
f_{*}^{n_{0} l}(\rho) \rightarrow \pi(\rho) \quad \text { as } l \rightarrow+\infty \text { in } L_{1}(\lambda) .
$$

We shall first prove the theorem for the spaces $C_{f, \varepsilon}^{(s-1)+1}$ instead of $C_{f, \varepsilon}^{s}$ and for $\|\cdot\|_{(s-1)+1}$ instead of $\|\cdot\|_{s}$

(a) follows for $C=\max \{2 \Theta, 2 T\}$ from remark 4.3(a), proposition 5.4 and remark 5.5(a).

Now, let $\rho_{l}=f_{*}^{n_{0} l}(1)$. By (a)

$$
\left\|\rho_{l}\right\|_{(r-2)+1} \leq C, \quad \text { for each } l \in \mathbb{N} \text {. }
$$

We want to prove that

$$
\pi(1) \in C_{f, E}^{(r-2)+1}
$$


Let us take a compact subset $K$ of $J \in \mathscr{J}$. We have

$$
\begin{aligned}
& \qquad\left.\left|\rho_{l}\right|_{K}\left|\leq C \cdot \sup _{K} \varphi_{\varepsilon}<+\infty, \quad\right| \rho_{l}^{(s)}\right|_{K} \mid \leq C \cdot \sup _{K} \varphi_{s}<+\infty, \\
& (s=1, \ldots, r-2) \text { and } \\
& \operatorname{Lip}\left(\left.\rho_{l}^{(r-2)}\right|_{K}\right) \leq C \cdot \sup _{K} \varphi_{r-1}<+\infty \quad l \in \mathbb{N} .
\end{aligned}
$$

Now applying the Ascoli-Arzela theorem we obtain by induction on $s$ that there exists a subsequence $\left\{\left.\rho_{l_{k}}\right|_{K}\right\}_{k \in \mathbb{N}}$ which converges in $C^{r-2}(K)$ to some $\rho_{K} \in C^{r-2}(K)$ and $\rho_{K}^{(r-2)}$ is Lipschitz on $K$. Since by (6.12) $\rho_{l}$ converges to $\pi(1)$ in $L_{1}(\lambda)$ we obtain $\left.\pi(1)\right|_{K}=\rho_{K}$ a.e. Hence since $K$ was arbitrary, some representative of $\pi(1)$ is of class $C^{r-2}$ and $\pi(1)^{(r-2)}$ is Lipschitz on compact subsets of $J \in \mathscr{F}$. It remains to estimate the norm of $\pi(1)$.

We can choose a diagonal subsequence of $\left\{\rho_{l_{k}}\right\}_{k \in \mathbb{N}}$ which converges pointwise to $\pi(1)$ with its derivatives up to order $r-2$. Hence $\|\pi(1)\|_{r-2} \leq C$. Now using the method of the proof of remark 4.3(b) (in particular $V_{k}$ ) we obtain:

$$
|\pi(1)|_{(r-2)+1} \leq C
$$

and hence

$$
\|\pi(1)\|_{(r-2)+1} \leq C .
$$

This ends the proof of (6.13).

In view of (6.2), (6.13) and the definition of $\pi$, we get

$$
\nu_{i, j} \in C_{f, \varepsilon}^{(r-2)+1} \text {. }
$$

Note the following

$$
\|\rho\|_{L_{1}} \leq \int \varphi_{\varepsilon} d \lambda \cdot\|\rho\|_{\varepsilon} \leq \int \varphi_{\varepsilon} d \lambda \cdot\|\rho\|_{(s-1)+1} .
$$

Hence we can prove the first part of (c) as follows:

$$
\begin{aligned}
\|\pi(\rho)\|_{(s-1)+1} & \leq \sum_{i, j} \int_{M_{i, j}}|\rho| d \lambda \cdot\left\|\nu_{i, j}\right\|_{(s-1)+1} \\
& \leq\|\rho\|_{L_{1}} \max _{i, j}\left\|\nu_{i, j}\right\|_{(s \sim 1)+1}
\end{aligned}
$$

This combined with $(6.15)$ proves that

(6.16) $\pi: C_{f, \varepsilon}^{(s-1)+1} \rightarrow C_{f, \varepsilon}^{(s-1)+1}$ is continuous and consequently the same is true for $\phi$.

Moreover from (6.9) and (a) for $s=1$ it follows that

$$
\left\|\phi^{n}\right\|_{(0)+1} \leq D \quad \text { for } n \in \mathbb{N}
$$

where $D=C+C\|\pi\|_{(0)+1}$. Write $\mathscr{B}=\mathrm{cl}_{L_{1}} \bigcup_{n=0}^{+\infty} \phi^{n}(R)$, where $R$ is a unit ball in $C_{f, \varepsilon}^{(0)+1}$ and notice that $\mathscr{B}$ is $\phi$-invariant, and by (6.17)

$$
\mathscr{B} \subset\left\{\rho \in C_{f, \varepsilon}^{(0)+1}:\|\rho\|_{(0)+1} \leq D\right\} .
$$

Hence by remark $4.3\left(\right.$ c) $\mathscr{B}$ is compact in $L_{1}(\lambda)$. Since $\left\|\phi^{n}\right\|_{L_{1}} \leq 2$, proposition 6.1 shows that the sequence of functions on $\mathscr{B} \subset L_{1}(\lambda),\left\{\left.\phi^{n}\right|_{\mathscr{B}}\right\}_{n \in \mathbb{N}}$, converges uniformly to zero. Therefore for some $\tilde{n} \in \mathbb{N},\left\|\phi^{\tilde{n}}(\rho)\right\|_{L_{1}}<\partial<1$ for $\rho \in \mathscr{B}$. Let us take $n \in \mathbb{N}$, 
$k \in \mathbb{N}$ and $\tilde{r}, 0 \leq \tilde{r}<\tilde{n}$ such that $n=k \cdot \tilde{n}+\tilde{r}$. Now we can estimate for $\rho \in R$ :

$$
\begin{aligned}
\left\|\phi^{n}(\rho)\right\|_{L_{1}} & =\left\|\phi^{k \tilde{n}+\tilde{r}}(\rho)\right\|_{L_{1}} \\
& \leq\left(\sup _{\tilde{\rho} \in \mathscr{B}}\left\|\phi^{\tilde{n}}(\tilde{\rho})\right\|_{L_{1}}\right)^{k} \cdot \sup _{\tilde{\rho} \in R}\left\|\phi^{\tilde{r}}(\tilde{\rho})\right\|_{L_{1}} \\
& \leq \partial^{k} \cdot 2 \leq \Gamma \cdot \gamma^{n},
\end{aligned}
$$

where $\gamma=\partial^{1 / \tilde{n}}$ and $\Gamma=2 / \gamma^{\tilde{n}}$, (notice that by (6.9), $\left\|\phi^{\tilde{n}}\right\|_{L_{1}} \leq 2$ ).

Finally, putting $\rho /\|\rho\|_{(0)+1}$ in (6.18) one obtains (d) for $\rho \in C_{f, \varepsilon}^{(0)+1}$.

(e) We shall first prove

$$
\lim _{n \rightarrow+\infty} \sup _{\rho \in R}\left\|\phi^{n}(\rho)\right\|_{\varepsilon}=0 .
$$

Let us take $\varepsilon^{\prime}>0$ and write for $\rho \in R$

$$
\left|\phi^{n}(\rho)\right|=\left|f_{*}^{n}(\rho-\pi(\rho))\right| \leq f_{*}^{n}\left(\varphi_{\varepsilon}\right) \cdot\|\rho-\pi(\rho)\|_{\varepsilon},
$$

and by proposition 5.4(a) we have

$$
\left|\phi^{n}(\rho)\right| \leq \Theta\left(\varphi_{0}+\vartheta^{n} \varphi_{\varepsilon}\right) \cdot\left(1+\|\pi\|_{\varepsilon}\right)
$$

Now we can find $\gamma>0$ and $n_{1} \in \mathbb{N}$ such that

$$
\frac{\left|\phi^{n}(\rho)(x)\right|}{\varphi_{\varepsilon}(x)}<\varepsilon^{\prime} \quad \text { for } x \in U_{\gamma}, n>n_{1} \text { and } \rho \in R .
$$

Besides (d) and (6.17) imply that $\phi^{n}(\rho)(x)$ converges uniformly to zero for $x \in I \backslash U_{\gamma}$ and $\rho \in R$ (notice that by (6.17) $\phi^{n}(\rho)^{\prime}(x)$ is bounded for $n \in \mathbb{N}, x \in I \backslash U_{\gamma}$ and $\rho \in R$ ). This enables us to find $n_{2} \in \mathbb{N}$ such that

$$
\frac{\phi^{n}(\rho)(x)}{\varphi_{\varepsilon}(x)}<\varepsilon^{\prime} \quad \text { for } \rho \in R, x \in I \backslash U_{v} \text { and } n>n_{2} \text {. }
$$

(6.20), (6.21) yield $\left\|\phi^{n}(\rho)\right\|_{\varepsilon} \leq \varepsilon^{\prime}$ for $n>\max \left\{n_{1}, n_{2}\right\}$ and $\rho \in R$, and this proves (6.19). We proceed to prove (e).

Recall the inequality from proposition 5.4(b) (compare remark 5.5(a)).

$$
\left|f_{*}^{k}(\rho)\right|_{1,(s-1)+1} \leq T\left(\tau^{k} \cdot|\rho|_{1,(s-1)+1}+\|\rho\|_{\varepsilon}\right) .
$$

Let $\mathbb{B}$ denote the unit ball in $C_{f, \varepsilon}^{(s-1)+1}$. Let us take $m \in \mathbb{N}$ such that $T \cdot\left\|\phi^{m}(\rho)\right\|_{\varepsilon}<\frac{1}{3}$ for $\rho \in R$ and let $k \in \mathbb{N}$ be such that $T \tau^{k} \cdot\left|\phi^{m}(\rho)\right|_{1,(s-1)+1}<\frac{1}{3}$ for $\rho \in \mathbb{B}$. ((c) and (6.19) enable us to do this). Now put $\phi^{m}(\rho)$ instead of $\rho$ in (6.22) and estimate

$$
\left|\phi^{k+m}(\rho)\right|_{1,(s-1)+1} \leq T\left(\tau^{k} \cdot\left|\phi^{m}(\rho)\right|_{1,(s-1)+1}+\left\|\phi^{m}(\rho)\right\|_{\varepsilon}\right)<\frac{2}{3}<1
$$

for $\rho \in \mathbb{B} \subset R$. Hence $\left\|\phi^{k+m}(\rho)\right\|_{(s-1)+1}<\frac{2}{3}$ for $\rho \in \mathbb{B}$ (one can assume that $T \geq 1$ ). The same reasoning as in (6.18) justifies the inequality in (e). Thus we have proved the theorem with $C_{f, \varepsilon}^{s}$ replaced by $C_{f, \varepsilon}^{(s-1)+1}$. Hence we shall deduce the theorem.

(a) follows from proposition 5.4. In view of the proved case of $(e), f_{*}^{n_{0} l}(1)$ converges to $\pi(1)$ in $C_{f, \varepsilon}^{(r-2)+1}$ as $l$ tends to $+\infty$. But since $f_{*}^{n_{0} l}(1) \in C_{f, \varepsilon}^{r-1}$ and $C_{f, \varepsilon}^{r-1}$ is closed in $C_{f, \varepsilon}^{(r-2)+1}$ and the respective norms coincide on $C_{f, \varepsilon}^{r-1}$, we have that $\pi(1)$ belongs to $C_{f, \varepsilon}^{r-1}$. Hence (b) (see the definition of $\pi$ ), (c) and (e) follow immediately.

Thus the proof is finished. 
Let $\lambda_{i} \in S^{1}$ be a generator of the cyclic group of order $k_{i}$. Let us define for $1 \leq i \leq p$, $1 \leq j \leq k_{i}$ the projections:

$$
\pi_{i, j}(\rho)=\frac{1}{k_{i}}\left(\sum_{l=1}^{k_{i}} \lambda_{i}^{-j l} \nu_{i, l}\right) \cdot\left(\sum_{n=1}^{k_{i}} \lambda_{i}^{j n} \cdot \int_{M_{i, n}} \rho d \lambda\right) .
$$

It is easy to check that $\pi_{i, j}$ is a projection onto the eigenspace of $f_{*}$ related to the eigenvalue $\lambda_{i}^{j}$. (It may happen that the lengths of two different cycles have a common divisor. Then certain eigenvalues will be repeated.)

Proposition 6.4. (a) $\sum_{i, j} \pi_{i, j}=\pi$.

(b) (spectral decomposition)

$$
f_{*}^{k}=\sum_{i, j}\left(\lambda_{i}^{j}\right)^{k} \pi_{i, j}+\phi^{k} \quad \text { for } k \in \mathbb{N} .
$$

(c) for all pairs $(i, j), \pi_{i, j^{\circ}} \phi^{\circ}=\phi^{\circ} \pi_{i, j}=0$ and $\pi_{i, j} \circ \pi_{i^{\prime}, j^{\prime}}=0$ if $(i, j) \neq\left(i^{\prime}, j^{\prime}\right)$.

Proof. (a) Assume that there is only one cycle, for the proof will be easier; (we shall omit the index $i$.)

$$
\sum_{j=1}^{k} \pi_{j}(\rho)=\frac{1}{k} \sum_{l=1}^{k} \sum_{n=1}^{k} \nu_{l} \cdot \int_{M_{n}} \rho d \lambda \cdot \Delta(n-l),
$$

where $\Delta(m)=\sum_{j=1}^{k} \lambda^{j m} \cdot \Delta(m)$ is equal to zero whenever $m \neq 0$ and it is equal to $k$ when $m=0$. Hence

$$
\sum_{j=1}^{k} \pi_{j}(\rho)=\sum_{i=1}^{k} \nu_{l} \int_{M_{1}} \rho d \lambda=\pi(\rho) .
$$

(b) follows from (a) and (6.9). Proof of (c) is trivial. One can check it using (6.3), the definition of operators and applying $\Delta$ as above.

\section{Lasota-Yorke type maps}

In this section we assume that $\left|f^{\prime}\right|>\gamma>1$ and $f$ on each component of $I \backslash A$ can be extended to the map of class $C^{r}(r \geq 2)$ on the closure of this component. Let us write

$$
\mathscr{J}=\{\vec{J}: J \text { is a component of } I \backslash \bar{B}\} .
$$

We define the spaces

$$
\begin{aligned}
& B V=\left\{\rho \in L_{1}(\lambda): \operatorname{var} \rho<+\infty\right\}, \\
& \widehat{B V}^{(r-2)+1}=\left\{\rho \in B V: \text { some representative of } \rho \text { is of class } C^{r-2} \text { and } \rho^{(r-2)}\right. \text { is } \\
& \quad \text { Lipschitz on all } J \in \mathscr{J}\}, \\
& \widehat{B V}^{r-1}=\left\{\rho \in B V: \text { some representative of } \rho \text { is of class } C^{r-1} \text { on all } J \in \mathscr{J}\right\} .
\end{aligned}
$$

For $\rho \in \widehat{B V}^{(r-2)+1}$ we define the semi-norms:

$$
\begin{aligned}
& |\rho|_{i}=\sup _{J \in \mathscr{I}} \sup _{J}\left|\rho^{(i)}\right| \quad\left(i=1,2, \ldots, r-2, \text { and } i=r-1 \text { if } \rho \in \widehat{B V}^{r-1}\right), \\
& |\rho|_{(r-2)+1}=\sup _{J \in \mathscr{J}} \operatorname{essup}_{J}\left|\rho^{(r-1)}\right| \\
& |\rho|_{1,(r-2)+1}=\max \left\{|\rho|_{1}, \ldots,|\rho|_{r-2},|\rho|_{(r-2)+1}\right\} .
\end{aligned}
$$

In the space $B V$ we have the norm

$$
\|\rho\|=\int_{I}|\rho| d \lambda+\operatorname{var} \rho,
$$


where var $\rho=\inf \left\{\operatorname{var} \rho^{\prime}: \rho^{\prime}=\rho\right.$ a.e. $\}$. Let us define the norm on $\widehat{B V}^{(r-2)+1}$ as

and the norm on $B V^{r-1}$

$$
\|\rho\|_{(r-2)+1}=\max \left\{\|\rho\|,|\rho|_{1,(r-2)+1}\right\}
$$

$$
\|\rho\|_{r-1}=\max \left\{\|\rho\|,|\rho|_{1}, \ldots,|\rho|_{r-1}\right\}
$$

Let us then define

$$
\begin{aligned}
B V^{(r-2)+1} & =\left\{\rho \in \widehat{B V}^{(r-2)+1}:\|\rho\|_{(r-2)+1}<+\infty\right\} \\
B V^{r-1} & =\left\{\rho \in \widehat{B V}^{r-1}:\|\rho\|_{r-1}<+\infty\right\} .
\end{aligned}
$$

According to M. Rychlik [8] there is a family of pairs $\left\{\left(K_{i, j}, \nu_{i, j}\right)\right\}_{1 \leq j \leq k_{i, 1}, i \leq p}$ such that the conditions (6.1)-(6.6) hold for the map $f$. Thus we can define basins $M_{i, j}$ and operators $\pi$ and $\phi$ as in $\S 6$. From [6] one can also deduce the following proposition.

Proposition 7.1. $\left\|\phi^{n}\right\|$ tends to zero exponentially as $n \rightarrow+\infty$.

We shall prove now the following theorem.

THEOREM 7.2. (a) $B V^{r-1}$ is an $f_{*}$-invariant subspace of $B V$.

(b) The densities $\nu_{i, j}$ belong to $B V^{r-1}$.

(c) $\left\|\phi^{n}\right\|_{r-1}$ converges to zero exponentially as $n \rightarrow+\infty$.

(d) For each density $\nu_{i, j}$ and for each $J \in \mathscr{J}$ either $\left.\nu_{i, j}\right|_{J} \equiv 0$, or $\left.\nu_{i, j}\right|_{J}$ is separated from zero (compare [3]).

Proof. (a) We have $f(\bar{B}) \subset \bar{B}$ and hence for each $J \in \mathscr{J}, f^{-1}(J) \subset \bigcup \mathscr{J}$. (a) now follows from the definition of $f_{*}$,

(c) We shall prove first that $\nu_{i, j} \in B V^{(r-2)+1}$. From lemma 1.2 it follows that

$$
\left|f_{*}^{k}(1)^{(s)}(x)\right| \leq f_{*}^{k}(1)(x) T(0, s, k)(x) \quad \text { for } x \in \hat{I} \text {. }
$$

Let us note that for some $C>0$

$$
T(l, s, k)(x) \leq C \quad \text { for } x \in I, s=1,2, \ldots, r-1, l \leq s, k \in \mathbb{N} .
$$

The proof of (7.2) is contained in the proofs of lemmas 4.1, 4.2. We will not repeat it.

Put $m=\prod_{i=1}^{p} k_{i}$. We have $f_{*}^{m} \circ \pi=\pi$. Hence by proposition 7.1 and (6.9) we have

(7.3) $f_{*}^{n m}(\rho)$ converges to $\pi(\rho)$ in $B V$ as $n$ tends to infinity.

Let us write $h_{n}=f_{*}^{n m}(1)$. By (7.1)-(7.3) there exists a constant $E>0$ such that $\left|h_{n}^{(s)}(x)\right| \leq E$ for $x \in \bigcup \mathscr{J}$ and $s=1, \ldots, r-1$. Let us fix $J \in \mathscr{F}$. From the Ascoli-Arzela theorem and (7.3) it follows by induction that $\left.\pi(1)\right|_{J}$ is of class $C^{(r-2)+1}$. Hence $\pi(1) \in B V^{(r-2)+1}$ and all $\nu_{i, j}$ belong to $B V^{(r-2)+1}$. From lemma 1.2 and (7.2) we can deduce that for some constant $F>0$

$$
\left|f_{*}^{k}(\rho)\right|_{1,(r-2)+1} \leq F\left(\gamma^{-k}|\rho|_{1,(r-2)+1}+\|\rho\|\right)
$$

for $k \in \mathbb{N}$ and $\rho \in B V^{(r-2)+1}, \gamma>1$. We omit the precise proof.

Let $B_{1}$ denote the unit ball in $B V^{(r-2)+1}$. We notice that by $(7.4),\left\|f_{*}^{k}\right\|_{(r-2)+1} \leq$ $\max \{2 F, D\}=D_{1}$. Hence by (6.9)

$$
\left\|\phi^{k}\right\|_{(r-2)+1} \leq D_{1}\left(1+\|\pi\|_{(r-2)+1}\right)=D_{2} \quad \text { for } k \in \mathbb{N} \text {, }
$$


where $D=\sup _{k \in \mathbb{N}}\left\|f_{*}^{k}\right\|<+\infty$. We also notice that since $\nu_{i, j} \in B V^{(r-2)+1},\|\pi\|_{(r-2)+1}<$ $+\infty$.

Take $n \in \mathbb{N}$ such that $F\left\|\phi^{n}\right\|<\frac{1}{3}$ and $k_{0}$ such that $F \gamma^{-k_{0}} D_{2}<\frac{1}{3}$. Now put $\phi^{n}(\rho), \rho \in$ $B_{1}$, in (7.4) in place of $\rho$ and we obtain

$$
\begin{aligned}
\left|\phi^{n+k_{0}}(\rho)\right|_{1,(r-2)+1} & \leq F\left(\gamma^{-k_{0}}\left|\phi^{n}(\rho)\right|_{1,(r-2)+1}+\left\|\phi^{n}(\rho)\right\|\right) \\
& \leq \frac{1}{3}+\frac{1}{3}=\frac{2}{3} .
\end{aligned}
$$

Hence $\left\|\phi^{n+k_{0}}\right\|_{(r-2)+1} \leq \frac{2}{3}<1$ and this yields

(7.5) $\left\|\phi^{k}\right\|_{(r-2)+1}$ converges to zero exponentially.

Therefore (7.3) now holds in $B V^{(r-2)+1}$ and, in particular, $h_{n}$ converges to $\pi(1)$ in $B V^{(r-2)+1}$. But $h_{n} \in B V^{r-1}, B V^{r-1} \subset B V^{(r-2)+1}$ are Banach spaces and norms $\|\cdot\|_{r-1}$ and $\|\cdot\|_{(r-2)+1}$ coincide on $B V^{r-1}$. Hence $\pi(1) \in B V^{r-1}$ and (b) holds. This gives us that $B V^{r-1}$ is invariant under $\phi$ and hence according to (7.5), (c) holds.

(d) Let us return to (7.1)-(7.2). We have that $\left|h_{n}^{\prime}\right| \leq C\left|h_{n}\right|$ and hence by (7.3)

$$
\left|\pi(1)^{\prime}(x)\right| \leq C \cdot \pi(1)(x) \quad \text { for } x \in J \in \mathscr{J} \text {. }
$$

We write $\nu=\pi(1)$. Thus we have $\left|(\ln \nu)^{\prime}\right| \leq C$ on $J \in \mathscr{J}$.

Fix $J \in \mathscr{J}$ and let $x_{0} \in J$. Then for $x \in J$

$$
\ln \nu\left(x_{0}\right)-C\left|x-x_{0}\right| \leq \ln \nu(x) \leq \ln \nu\left(x_{0}\right)+C\left|x-x_{0}\right|
$$

and

$$
\nu\left(x_{0}\right) e^{-C\left|x-x_{0}\right|} \leq \nu(x) \leq \nu\left(x_{0}\right) e^{C\left|x-x_{0}\right|}
$$

Hence (d) follows.

Finally in this section we shall define the Banach space $B V_{1}=\{\rho \in B V$ : the continuous part of some representative of $\rho$ is Lipschitz $\}$. Since a function of bounded variation can be decomposed into the sum of a step function and a continuous function, this definition makes sense. Define the norm

$$
\|\rho\|_{1}=\max \{\|\rho\|, \text { Lip cp } \rho\},
$$

where cp $\rho$ denotes the continuous part of $\rho$. Notice that Lip cp $\rho=\operatorname{essup}\left|\rho^{\prime}\right|$ and recall that a function of bounded variation is almost everywhere differentiable.

THEOREM 7.3. Let $r=2$.

(a) $B V_{1}$ is an $f_{*}$-invariant subspace of $B V$.

(b) The densities $\nu_{i, j}$ belong to $B V_{1}$.

(c) $\left\|\phi^{n}\right\|_{1}$ converges exponentially to zero as $n \rightarrow+\infty$.

We shall not prove this theorem because the proof is almost the same as the proof of theorem 7.2. We remark only that if a sequence $\rho_{n}$ converges to $\rho$ in the norm $\|\cdot\|$ then its continuous part and its step part converge uniformly to the continuous part and step part of $\rho$ respectively. This enables one to repeat the proof of theorem $7.2(\mathrm{~b})$, (c).

\section{REFERENCES}

[1] F. Hofbauer \& G. Keller. Ergodic properties of invariant measures for piecewise monotonic transformations. Math. Z. 180 (1982), 119-140. 
[2] F. Hofbauer \& G. Keller. Equilibrium states for piecewise monotonic transformations. Erg. Th. \& Dynam. Sys. 2 (1982), 23-43.

[3] Z. S. Kowalski. Invariant measure of piecewise monotonic transformation has a positive lower bound on its support. Bull. Acad. Polon. Sci., Ser. Sci. Math. 27 (1979), 53-57.

[4] K. Krzyżewski. Some results on expanding mappings. Asterisque 50 (1977), 205-218.

[5] A. Lasota \& J. Yorke. On the existence of invariant measures for piecewise monotonic transformations. Trans. Amer. Math. Soc. 186 (1973), $481-488$.

[6] M. Misiurewicz. Absolutely continuous measures for certain maps of an interval. Inst. Hautes Études Sci. Publ. Math. 53 (1981), 17-51.

[7] A. J. Ognev. Metric properties of some class of maps of an interval. Mat. Zametki 30 No 5 (1981) 723-736. (In Russian.)

[8] M. Rychlik. Bounded variation and invariant measures. Preprint, to appear in Studia Mathematica.

[9] R. Sacksteder. The measures invariant under an expanding map. In Springer Lecture Notes in Maths 392 (1974).

[10] W. Szlenk. Some dynamical properties of certain differentiable mappings of an interval, Part I-Boletin de la Sociedad Mathematica Mexicana, 24 No 2 (1979), 57-82; Part II, Ergodic Theory and Dynamical Systems II (Proceedings Special Year, Maryland 1979-80). Birkhäuser.

[11] K. Ziemian. Thesis, Warsaw University. 